\title{
UNIFORM AND OPTIMAL ERROR ESTIMATES OF AN EXPONENTIAL WAVE INTEGRATOR SINE PSEUDOSPECTRAL METHOD FOR THE NONLINEAR SCHRÖDINGER EQUATION WITH WAVE OPERATOR*
}

\author{
WEIZHU $\mathrm{BAO}^{\dagger}$ AND YONGYONG $\mathrm{CAI}^{\ddagger}$
}

\begin{abstract}
We propose an exponential wave integrator sine pseudospectral (EWI-SP) method for the nonlinear Schrödinger equation (NLS) with wave operator (NLSW), and carry out rigorous error analysis. The NLSW is NLS perturbed by the wave operator with strength described by a dimensionless parameter $\varepsilon \in(0,1]$. As $\varepsilon \rightarrow 0^{+}$, the NLSW converges to the NLS and for the small perturbation, i.e. $0<\varepsilon \ll 1$, the solution of the NLSW differs from that of the NLS with a function oscillating in time with $O\left(\varepsilon^{2}\right)$-wavelength at $O\left(\varepsilon^{2}\right)$ and $O\left(\varepsilon^{4}\right)$ amplitudes for ill-prepared and wellprepared initial data, respectively. This rapid oscillation in time brings significant difficulties in designing and analyzing numerical methods with error bounds uniformly in $\varepsilon$. In this work, we show that the proposed EWI-SP possesses the optimal uniform error bounds at $O\left(\tau^{2}\right)$ and $O(\tau)$ in $\tau$ (time step) for well-prepared initial data and ill-prepared initial data, respectively, and spectral accuracy in $h$ (mesh size) for the both cases, in the $L^{2}$ and semi- $H^{1}$ norms. This result significantly improves the error bounds of the finite difference methods for the NLSW. Our approach involves a careful study of the error propagation, cut-off of the nonlinearity and the energy method. Numerical examples are provided to confirm our theoretical analysis.
\end{abstract}

Key words. nonlinear Schrödinger equation with wave operator, error estimates, exponential wave integrator, sine pseudospectral method

AMS subject classifications. 35Q55, 65M12, 65M15, 65M70, 81-08

1. Introduction. In this paper, we consider the nonlinear Schrödinger equation with wave operator (NLSW) in $d(d=1,2,3)$ dimensions as $4,6,8,11,22,25,29,31]$

$$
\begin{cases}i \partial_{t} \psi(\mathbf{x}, t)-\varepsilon^{2} \partial_{t t} \psi(\mathbf{x}, t)+\nabla^{2} \psi(\mathbf{x}, t)+f\left(|\psi(\mathbf{x}, t)|^{2}\right) \psi(\mathbf{x}, t)=0, & \mathbf{x} \in \mathbb{R}^{d}, t>0, \\ \psi(\mathbf{x}, 0)=\psi_{0}(\mathbf{x}), & \partial_{t} \psi(\mathbf{x}, 0)=\psi_{1}^{\varepsilon}(\mathbf{x}),\end{cases}
$$

where $\psi:=\psi(\mathbf{x}, t)$ is a complex function, $\mathbf{x}$ is the spatial variable, $t$ is the time, $0<\varepsilon \leq 1$ is a dimensionless parameter, $f:[0,+\infty) \rightarrow \mathbb{R}$ is a real-valued function, and $\nabla^{2}=\Delta$ is the Laplace operator in $d$ dimensional space. In this paper, we will consider $\psi_{1}^{\varepsilon}$ to be $O(1)$ w.r.t $\varepsilon$.

The NLSW has different physical applications, including the nonrelativistic limit of the KleinGordon equation [22, 25, 29], the Langmuir wave envelope approximation in plasma [8, 11, and the modulated planar pulse approximation of the sine-Gordon equation for light bullets [6, 31].

As proven by [8,22, 25, 29], when $\varepsilon \rightarrow 0^{+}$, the NLSW (1.1) converges to the standard nonlinear Schrödinger equation (NLS),

$$
\begin{cases}i \partial_{t} \psi(\mathbf{x}, t)+\nabla^{2} \psi(\mathbf{x}, t)+f\left(|\psi(\mathbf{x}, t)|^{2}\right) \psi(\mathbf{x}, t)=0, & \mathbf{x} \in \mathbb{R}^{d}, t>0, \\ \psi(\mathbf{x}, 0)=\psi_{0}(\mathbf{x}), & \mathbf{x} \in \mathbb{R}^{d} .\end{cases}
$$

${ }^{*}$ This work was supported by the Singapore A*STAR SERC PSF-Grant 1321202067 (W. Bao) and the KI-NET at the Center for Scientific Computation \& Math Modeling (CSCAMM) in the University of Maryland (Y. Cai).

${ }^{\dagger}$ Department of Mathematics and Center for Computational Science and Engineering, National University of Singapore, Singapore 119076 (bao@math.nus.edu.sg, URL: http://www.math.nus.edu.sg/ bao/)

${ }^{\ddagger}$ Beijing Computational Science Research Center, Beijing 100084, P. R. China; and Department of Mathematics, National University of Singapore, Singapore 119076 (chainrules@gmail.com). Current address: Center of Scientific Computation and Mathematical Modeling (CSCAMM), University of Maryland, College Park, MD 20742, USA 
Due to the wave operator, the solution of the NLSW (1.1) differs from the solution of the NLS (1.2) with a function oscillating in time $t$ with $O\left(\varepsilon^{2}\right)$ wavelength. Indeed, to measure the difference between the NLSW (1.1) and the NLS (1.2), we can write the initial velocity $\psi_{1}^{\varepsilon}$ for the NLSW (1.1) as

$$
\psi_{1}^{\varepsilon}(\mathbf{x})=i\left(\nabla^{2} \psi_{0}(\mathbf{x})+f\left(\left|\psi_{0}(\mathbf{x})\right|^{2}\right) \psi_{0}(\mathbf{x})\right)+\varepsilon^{\alpha} \omega^{\varepsilon}(\mathbf{x}), \quad \alpha \geq 0,
$$

where $i\left(\nabla^{2} \psi_{0}(\mathbf{x})+f\left(\left|\psi_{0}(\mathbf{x})\right|^{2}\right) \psi_{0}(\mathbf{x})\right)$ corresponds to the initial velocity for the NLS (1.2). Then we have the following asymptotic expansion for the solution $\psi(\mathbf{x}, t)$ of the NLSW (1.1) as $\varepsilon \rightarrow 0^{+}$(cf. 4])

$$
\begin{aligned}
\psi(\mathbf{x}, t)= & \psi^{s}(\mathbf{x}, t)+\varepsilon^{2}\{\text { terms without oscillation }\} \\
& +\varepsilon^{2+\min \{\alpha, 2\}} \Psi\left(\mathbf{x}, t / \varepsilon^{2}\right)+\text { higher order terms with oscillation, }
\end{aligned}
$$

where $\psi^{s}:=\psi^{s}(\mathbf{x}, t)$ satisfies the NLS (1.2).

Based on this asymptotic expansion, we can make assumptions (A) and (B) (cf. section 2.2) on the NLSW. Furthermore, from (1.4), we identify two cases for the imposed initial data (1.3), i.e., the well-prepared initial data when $\alpha \geq 2$ and the ill-prepared initial data when $0 \leq \alpha<2$.

Various numerical methods have been developed in the literatures for the NLS, including the time-splitting pseudospectral method [3, 7, 9, 17, 21, 23, 24, 27, finite difference method [1, 3, 10, 15], etc. Meanwhile, there are few numerical methods for the NLSW and conservative finite difference methods 11, 16, 30 are the most popular ones. In our recent work [4, both the conservative CrankNicolson finite difference scheme and the semi-implicit finite difference scheme have been analyzed for NLSW. We have proved the uniform $l^{2}$ and semi- $H^{1}$ error estimates of these two schemes w.r.t. $\varepsilon \in(0,1]$, at the order of $O\left(h^{2}+\tau\right)$ for well-prepared initial data and $O\left(h^{2}+\tau^{2 / 3}\right)$ for ill-prepared initial data, in all dimensions $(d=1,2,3)$, with mesh size $h$ and time step $\tau$. However, the uniform convergence rates are not optimal in $\tau$. Here, we propose an exponential wave integrator sine pseudospectral (EWISP) method with uniform spectral accuracy in space, and the method has the optimal uniform error bounds in time at the order $O\left(\tau^{2}\right)$ for well-prepared initial data and $O(\tau)$ for ill-prepared initial data. EWI-SP greatly improves the uniform convergence rate compared to the finite difference methods, and the uniform error bounds are optimal for the well-prepared initial data case. In fact, the exponential wave integrator has been widely used in solving highly oscillatory PDE [5] and ODE problems [14, 18, and the main advantage of EWI-SP is that only second order derivatives in time are involved, which is the key point in our analysis to get the optimal and uniform error bounds. Other techniques of the analysis include the cut-off of the nonlinearity, energy method, and recursion properties of the scheme.

This paper is organized as follows. In section 2, we introduce EWI-SP method and our main results. Section 3 is devoted to the error estimates of EWI-SP in the case of well-prepared initial data. In section 4, ill-prepared initial data case is considered. Numerical results are reported in section 5 to confirm the error estimates. Then some conclusions are drawn in section 6 . Throughout the paper, $C$ will denote a generic constant independent of $\varepsilon$, mesh size $h$ and time step $\tau$, and we use the notation $A \lesssim B$ to mean that there exists a generic constant $C$ which is independent of $\varepsilon$, time step $\tau$ and mesh size $h$ such that $|A| \leq C B$.

2. Exponential-wave-integrator sine pseudospectral method and results. In practical computation, the NLSW (1.1) is usually truncated on a bounded interval $\Omega=(a, b)$ in $1 \mathrm{D}(d=1)$, or a bounded rectangle $\Omega=(a, b) \times(c, d)$ in $2 \mathrm{D}(d=2)$ or a bounded box $\Omega=(a, b) \times(c, d) \times(e, f)$ in 3D $(d=3)$, with zero Dirichlet boundary condition. This truncation is accurate as long as the solution of (1.1) stays localized. For the simplicity of notation, we only deal with the case in 1D, i.e. $d=1$ and $\Omega=(a, b)$. Extensions to $2 \mathrm{D}$ and $3 \mathrm{D}$ are straightforward, and the error estimates in $L^{2}$-norm and semi- $H^{1}$ norm are the same in 2D and 3D (cf. Remark 4.1). In 1D, NLSW (1.1) is truncated on the 
interval $\Omega=(a, b)$ as

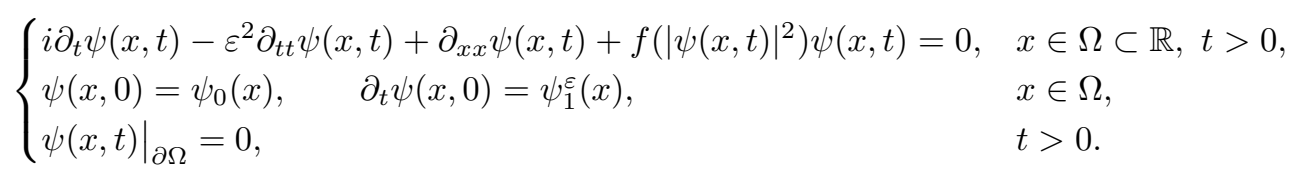

As $\varepsilon \rightarrow 0^{+}$, the solution of equation (2.1) will converge to the solution of the corresponding NLS [4, $, 8,25,29$.

$$
\begin{cases}i \partial_{t} \psi^{s}(x, t)+\partial_{x x} \psi^{s}(x, t)+f\left(\left|\psi^{s}(x, t)\right|^{2}\right) \psi^{s}(x, t)=0, & x \in \Omega \subset \mathbb{R}, t>0, \\ \psi^{s}(x, 0)=\psi_{0}(x), & x \in \Omega, \\ \left.\psi^{s}(x, t)\right|_{\partial \Omega}=0, & t>0 .\end{cases}
$$

In the spirit of (1.3), we assume the initial data satisfy the condition

$$
\psi_{1}^{\varepsilon}(x)=\psi_{1}(x)+\varepsilon^{\alpha} \omega^{\varepsilon}(x), \quad x \in \Omega,
$$

where $\psi_{1}(x)=i\left(\partial_{x x} \psi_{0}(x)+f\left(\left|\psi_{0}(x)\right|^{2}\right) \psi_{0}(x)\right)$ is the value of $\left.\partial_{t} \psi^{s}(x, t)\right|_{t=0}$ with $\psi^{s}(x, t)$ being the solution of the limiting NLS (2.2), $\omega^{\varepsilon}$ is uniformly bounded in $H_{0}^{1} \cap H^{2}$ (w.r.t. $\varepsilon$ ) and satisfies $\liminf \operatorname{in}_{\varepsilon \rightarrow 0^{+}}\left\|\omega^{\varepsilon}\right\|_{H^{2}}>0$ and $\alpha \geq 0$ is a parameter describing the compatibility of the initial data with respect to the limiting NLS (2.2).

2.1. EWI-SP method. For simplicity of notations, we define $\alpha^{*}$ as

$$
\alpha^{*}=\min \{\alpha, 2\} .
$$

We also denote the nonlinear term $f\left(|z|^{2}\right) z$ as

$$
\mathcal{F}(z)=f\left(|z|^{2}\right) z, \quad \forall z \in \mathbb{C} .
$$

Choose time step $\tau:=\Delta t$ and denote time steps as $t_{n}:=n \tau$ for $n=0,1,2, \ldots$; choose mesh size $\Delta x:=\frac{b-a}{M}$ with $M$ being a positive integer and denote $h:=\Delta x$ and grid points as

$$
x_{j}:=a+j \Delta x, \quad j=0,1, \ldots, M .
$$

Define the index sets

$$
\mathcal{T}_{M}=\{j \mid j=1,2, \ldots, M-1\}, \quad \mathcal{T}_{M}^{0}=\{j \mid j=0,1,2 \ldots, M\} .
$$

and denote

$$
\begin{aligned}
& X_{M}=\operatorname{span}\left\{\Phi_{l}(x)=\sin \left(\mu_{l}(x-a)\right), \quad \mu_{l}=\frac{\pi l}{b-a}, \quad x \in \Omega, l \in \mathcal{T}_{M}\right\}, \\
& Y_{M}=\left\{v=\left(v_{0}, v_{1}, \ldots, v_{M}\right)^{T} \in \mathbb{C}^{M+1} \mid v_{0}=v_{M}=0\right\} .
\end{aligned}
$$

We define the finite difference operators as usual, for $\psi\left(x_{j}, t_{n}\right)\left(j \in \mathcal{T}_{M}^{0}, n \geq 1\right)$,

$$
\delta_{x}^{+} \psi\left(x_{j}, t_{n}\right)=\frac{1}{h}\left(\psi\left(x_{j+1}, t_{n}\right)-\psi\left(x_{j}, t_{n}\right)\right), \quad \delta_{t}^{-} \psi\left(x_{j}, t_{n}\right)=\frac{1}{\tau}\left(\psi\left(x_{j}, t_{n}\right)-\psi\left(x_{j}, t_{n-1}\right)\right) .
$$


The exponential wave integrator sine spectral method of NLSW (2.1) is to apply sine spectral method for spatial discretization and exponential wave integrator for time. For any function $\psi(x) \in$ $L^{2}(\Omega), \phi(x) \in C_{0}(\bar{\Omega})$, and vector $\phi=\left(\phi_{0}, \phi_{1}, \ldots, \phi_{M}\right)^{T} \in Y_{M}$, let $P_{M}: L^{2}(\Omega) \rightarrow X_{M}$ be the standard $L^{2}$ projection onto $X_{M}$ and $I_{M}: C_{0}(\bar{\Omega}) \rightarrow X_{M}$ and $I_{M}: Y_{M} \rightarrow X_{M}$ be the standard sine interpolation operator as

$$
\left(P_{M} \psi\right)(x)=\sum_{l=1}^{M-1} \hat{\psi}_{l} \sin \left(\mu_{l}(x-a)\right), \quad\left(I_{M} \phi\right)(x)=\sum_{l=1}^{M-1} \tilde{\phi}_{l} \sin \left(\mu_{l}(x-a)\right), \quad x \in \bar{\Omega}=[a, b],
$$

and the coefficients are given by

$$
\hat{\psi}_{l}=\frac{2}{b-a} \int_{a}^{b} \psi(x) \sin \left(\mu_{l}(x-a)\right) d x, \quad \tilde{\phi}_{l}=\frac{2}{M} \sum_{j=1}^{M-1} \phi_{j} \sin (j l \pi / M), \quad l \in \mathcal{T}_{M},
$$

where $\phi_{j}=\phi\left(x_{j}\right)$ when $\phi$ is a function instead of a vector.

The sine spectral discretization for equation (2.1) becomes:

Find

$$
\psi_{M}:=\psi_{M}(x, t)=\sum_{l=1}^{M-1} \widehat{\psi}_{l}(t) \sin \left(\mu_{l}(x-a)\right), \quad x \in \Omega, t \geq 0
$$

such that

$$
\left(i \partial_{t}-\varepsilon^{2} \partial_{t t}\right) \psi_{M}(x, t)+\Delta \psi_{M}(x, t)+P_{M}\left(f\left(\left|\psi_{M}\right|^{2}\right) \psi_{M}\right)=0, \quad x \in \Omega, t>0 .
$$

Substituting (2.10) into (2.11) and making use of the fact that $\Phi_{l}(x)=\sin \left(\mu_{l}(x-a)\right)$ (2.6) are orthogonal to each other, we have

$$
-\varepsilon^{2} \frac{d^{2}}{d t^{2}} \widehat{\psi}_{l}(t)+i \frac{d}{d t} \widehat{\psi}_{l}(t)-\left|\mu_{l}\right|^{2} \widehat{\psi}_{l}(t)+\left(\widehat{\mathcal{F}\left(\psi_{M}\right)}\right)_{l}=0, \quad l \in \mathcal{T}_{M}, t>0 .
$$

Then, a numerical method can be designed by properly treating the above second order ODEs (2.12) 5, 14, 18, e.g. solving (2.12) via variation of constant formula and approximating the convolution term in a good manner. Now, let us state our approach in detail. For each $l \in \mathcal{T}_{M}$, around time $t_{n}=n \tau$ $(n \geq 0)$, we reformulate the above ODE as

$$
-\varepsilon^{2} \frac{d^{2}}{d t^{2}} \widehat{\psi}_{l}\left(t_{n}+s\right)+i \frac{d}{d t} \widehat{\psi}_{l}\left(t_{n}+s\right)-\left|\mu_{l}\right|^{2} \widehat{\psi}_{l}\left(t_{n}+s\right)+f_{l}^{n}(s)=0, \quad s \in \mathbb{R},
$$

where

$$
f_{l}^{n}(s)=\left(\widehat{\mathcal{F}\left(\psi_{M}\right)}\right)_{l}\left(t_{n}+s\right) .
$$

For $l \in \mathcal{T}_{M}$ and $s \in \mathbb{R}$, we denote

$$
\begin{aligned}
& \beta_{l}^{+}=\frac{1+\sqrt{1+4 \varepsilon^{2}\left|\mu_{l}\right|^{2}}}{2 \varepsilon^{2}}=O\left(\frac{1}{\varepsilon^{2}}\right), \quad \beta_{l}^{-}=\frac{1-\sqrt{1+4 \varepsilon^{2}\left|\mu_{l}\right|^{2}}}{2 \varepsilon^{2}}=\frac{-2\left|\mu_{l}\right|^{2}}{1+\sqrt{1+4 \varepsilon^{2}\left|\mu_{l}\right|^{2}}} O(1), \\
& \beta_{l}=\beta_{l}^{+}-\beta_{l}^{-}=\frac{\sqrt{1+4 \varepsilon^{2}\left|\mu_{l}\right|^{2}}}{\varepsilon^{2}}=O\left(\frac{1}{\varepsilon^{2}}\right), \quad \kappa_{l}(s)=e^{i s \beta_{l}^{+}}-e^{i s \beta_{l}^{-}}=O(1) .
\end{aligned}
$$


Solving the above second order ODE (2.13), e.g., using the variation-of-constant formula, the solution can be written as follows, for any $s \in \mathbb{R}$,

$$
\widehat{\psi}_{l}\left(t_{n}+s\right)=\gamma_{l}^{n} e^{i \beta_{l}^{+} s}+\nu_{l}^{n} e^{i \beta_{l}^{-} s}-\frac{i}{\varepsilon^{2} \beta_{l}} \int_{0}^{s} f_{l}^{n}\left(s_{1}\right) \kappa_{l}\left(s-s_{1}\right) d s_{1},
$$

with

$$
\gamma_{l}^{n}=-\frac{\beta_{l}^{-} \widehat{\psi}_{l}\left(t_{n}\right)+i \partial_{t} \widehat{\psi}_{l}\left(t_{n}\right)}{\beta_{l}}, \quad \nu_{l}^{n}=\frac{\beta_{l}^{+} \widehat{\psi}_{l}\left(t_{n}\right)+i \partial_{t} \widehat{\psi}_{l}\left(t_{n}\right)}{\beta_{l}} .
$$

Using the formula (2.16) and (2.17), we are going to determine the suitable approximations of $\widehat{\psi}_{l}$ at different time steps. For $n=0$, let $s=\tau$ in (2.16), then we get

$$
\widehat{\psi}_{l}(\tau)=\gamma_{l}^{0} e^{i \beta_{l}^{+} \tau}+\nu_{l}^{0} e^{i \beta_{l}^{-} \tau}-\frac{i}{\varepsilon^{2} \beta_{l}} \int_{0}^{\tau} f_{l}^{0}(s) \kappa_{l}(\tau-s) d s,
$$

For $n \geq 1$, choosing $s= \pm \tau$ in (2.16), and eliminating the $\partial_{t} \widehat{\psi}_{l}\left(t_{n}\right)$ term, we have

$$
\begin{aligned}
\widehat{\psi_{l}}\left(t_{n+1}\right)= & -e^{\frac{i \tau}{\varepsilon^{2}}} \widehat{\psi}_{l}\left(t_{n-1}\right)+2 e^{\frac{i \tau}{2 \varepsilon^{2}}} \cos \left(\tau \beta_{l} / 2\right) \widehat{\psi}_{l}\left(t_{n}\right)-\frac{i}{\varepsilon^{2} \beta_{l}} \int_{0}^{\tau} f_{l}^{n}(s) \kappa_{l}(\tau-s) d s \\
& +i \frac{e^{i \tau / \varepsilon^{2}}}{\varepsilon^{2} \beta_{l}} \int_{0}^{\tau} f_{l}^{n-1}(s) \kappa_{l}(-s) d s .
\end{aligned}
$$

For convenience, we recall the definition of 'sinc' function as

$$
\operatorname{sinc}(s)=\frac{\sin (s)}{s}, \quad \text { for } \quad s \neq 0, \text { and } \operatorname{sinc}(0)=1,
$$

and introduce the following notations for $l \in \mathcal{T}_{M}$,

$$
\sigma_{l}^{+}(s)=e^{i s \beta_{l}^{+} / 2} \operatorname{sinc}\left(s \beta_{l}^{+} / 2\right), \quad \sigma_{l}^{-}(s)=e^{i s \beta_{l}^{-} / 2} \operatorname{sinc}\left(s \beta_{l}^{-} / 2\right), \quad s \in \mathbb{R} .
$$

We approximate the integrals in (2.18) and (2.19) as follows:

$$
\begin{aligned}
& \int_{0}^{\tau} f_{l}^{0}(s) \kappa_{l}(\tau-s) d s \approx \int_{0}^{\tau}\left(f_{l}^{0}(0)+s \partial_{t} f_{l}^{0}(0)\right) \kappa(\tau-s) d s \\
& =\tau f_{l}^{0}(0)\left(\sigma_{l}^{+}(\tau)-\sigma_{l}^{-}(\tau)\right)+i \tau \partial_{t} f_{l}^{0}(0)\left(\frac{1-\sigma_{l}^{+}(\tau)}{\beta_{l}^{+}}-\frac{1-\sigma_{l}^{-}(\tau)}{\beta_{l}^{-}}\right), \\
& \int_{0}^{\tau} f_{l}^{0}(s) \kappa_{l}(-s) d s \approx \tau f_{l}^{0}(0)\left(\overline{\sigma_{l}^{+}(\tau)}-\overline{\sigma_{l}^{-}(\tau)}\right)+i \tau \partial_{t} f_{l}^{0}(0)\left(\frac{1-\sigma_{l}^{+}(\tau)}{\beta_{l}^{+} e^{i \tau \beta_{l}^{+}}}-\frac{1-\sigma_{l}^{-}(\tau)}{\beta_{l}^{-} e^{i \tau \beta_{l}^{-}}}\right),
\end{aligned}
$$

where $\bar{c}$ denotes the complex conjugate of $c$ and $\partial_{t} f_{l}^{0}(0)$ can be computed exactly since $\partial_{t} \psi(t=0)$ is known using the initial data. For $n \geq 1$, we use the similar approximation as above and approximate $\partial_{t} f_{l}^{n}(0)$ by finite difference $\delta_{t}^{-} f_{l}^{n}(0)$,

$$
\begin{aligned}
\int_{0}^{\tau} f_{l}^{n}(s) \kappa_{l}(\tau-s) d s & \approx \tau f_{l}^{n}(0)\left(\sigma_{l}^{+}(\tau)-\sigma_{l}^{-}(\tau)\right)+i \tau \delta_{t}^{-} f_{l}^{n}(0)\left(\frac{1-\sigma_{l}^{+}(\tau)}{\beta_{l}^{+}}-\frac{1-\sigma_{l}^{-}(\tau)}{\beta_{l}^{-}}\right), \\
\int_{0}^{\tau} f_{l}^{n}(s) \kappa_{l}(-s) d s & \approx \tau f_{l}^{n}(0)\left(\overline{\sigma_{l}^{+}(\tau)}-\overline{\sigma_{l}^{-}(\tau)}\right)+i \tau \delta_{t}^{-} f_{l}^{n}(0)\left(\frac{1-\sigma_{l}^{+}(\tau)}{\beta_{l}^{+} e^{i \tau \beta_{l}^{+}}}-\frac{1-\sigma_{l}^{-}(\tau)}{\beta_{l}^{-} e^{i \tau \beta_{l}^{-}}}\right) .
\end{aligned}
$$


For convenience of notations, we denote $\mathcal{D}\left(\phi\left(x, t_{n}\right)\right)$ for $\phi(x, t)$ as

$$
\mathcal{D}(\phi(0))=\left.\frac{d}{d t} \mathcal{F}(\phi(t))\right|_{t=0}, \quad \mathcal{D}\left(\phi\left(t_{n}\right)\right)=\delta_{t}^{-} \mathcal{F}\left(\phi\left(t_{n}\right)\right), \quad n \geq 1 .
$$

Thus, sine spectral method for solving (2.1) can be derived as follows. For $n \geq 0$, let $\psi_{M}^{n}(x)$ be the approximations of $\psi_{M}\left(x, t_{n}\right)$. Choose $\psi_{M}^{0}=P_{M}\left(\psi_{0}\right)$, then $\psi_{M}^{n+1}(x)$ for $n \geq 0$ is given by

$$
\psi_{M}^{n+1}(x)=\sum_{l=1}^{M-1}\left(\widehat{\psi_{M}^{n+1}}\right)_{l} \sin \left(\mu_{l}(x-a)\right), \quad x \in \Omega, \quad n=0,1, \ldots,
$$

where the sine transform coefficients can be obtained via the following formula

$$
\begin{aligned}
\left(\widehat{\psi_{M}^{1}}\right)_{l} & \left.=c_{l}^{0}\left(\widehat{\psi_{M}^{0}}\right)_{l}+d_{l}^{0}\left(\widehat{\psi_{1}^{\varepsilon}}\right)_{l}+p_{l}\left(\widehat{\mathcal{F}\left(\psi_{M}^{0}\right)}\right)_{l}+q_{l}\left(\widehat{\mathcal{D}\left(\psi_{M}^{0}\right.}\right)\right)_{l}, \quad \text { and for } n \geq 1 \text { as } \\
\left(\widehat{\psi_{M}^{n+1}}\right)_{l} & \left.\left.\left.=c_{l}\left(\widehat{\psi_{M}^{n-1}}\right)_{l}+d_{l}\left(\widehat{\psi_{M}^{n}}\right)_{l}+p_{l}\left(\widehat{\mathcal{F}\left(\psi_{M}^{n}\right.}\right)\right)_{l}+q_{l}\left(\widehat{\mathcal{D}\left(\psi_{M}^{n}\right.}\right)\right)_{l}-p_{l}^{*}\left(\widehat{\mathcal{F}\left(\psi_{M}^{n-1}\right.}\right)\right)_{l}-q_{l}^{*}\left(\widehat{\left.\mathcal{D}\left(\psi_{M}^{n-1}\right)\right)_{l}},\right.
\end{aligned}
$$

where $\mathcal{D}\left(\psi_{M}^{n}\right)(n \geq 0)$ is given by (2.23) as

$$
\mathcal{D}\left(\psi_{M}^{0}\right)=G\left(\psi_{M}^{0}\right) P_{M}\left(\psi_{1}^{\varepsilon}\right)+H\left(\psi_{M}^{0}\right) \overline{P_{M}\left(\psi_{1}^{\varepsilon}\right)}, \quad \mathcal{D}\left(\psi_{M}^{n}\right)=\delta_{t}^{-} \mathcal{F}\left(\psi_{M}^{n}\right), \quad n \geq 1,
$$

with

$$
\begin{aligned}
& G(z)=f\left(|z|^{2}\right)+f^{\prime}\left(|z|^{2}\right) \cdot|z|^{2}, \quad H(z)=f^{\prime}\left(|z|^{2}\right) z^{2}, \quad \forall z \in \mathbb{C}, \\
& c_{l}^{0}=\frac{\beta_{l}^{+} e^{i \tau \beta_{l}^{-}}-\beta_{l}^{-} e^{i \tau \beta_{l}^{+}}}{\beta_{l}}, \quad d_{l}^{0}=-i \tau e^{i \frac{\tau}{2 \varepsilon^{2}}} \operatorname{sinc}\left(\tau \frac{\beta_{l}}{2}\right), \quad p_{l}=\frac{-i \tau}{\varepsilon^{2} \beta_{l}}\left(\sigma_{l}^{+}(\tau)-\sigma_{l}^{-}(\tau)\right), \\
& \left.q_{l}=\frac{\tau}{\varepsilon^{2} \beta_{l}}\left(\frac{1-\sigma_{l}^{+}(\tau)}{\beta_{l}^{+}}-\frac{1-\sigma_{l}^{-}(\tau)}{\beta_{l}^{-}}\right), \quad p_{l}^{*}=\frac{-i \tau e^{\frac{i \tau}{\varepsilon^{2}}}}{\varepsilon^{2} \beta_{l}} \overline{\left(\sigma_{l}^{+}(\tau)\right.}-\overline{\sigma_{l}^{-}(\tau)}\right), \\
& q_{l}^{*}=\frac{\tau e^{\frac{i \tau}{\varepsilon^{2}}}}{\varepsilon^{2} \beta_{l}}\left(\frac{1-\sigma_{l}^{+}(\tau)}{e^{i \tau \beta_{l}^{+}} \beta_{l}^{+}}-\frac{1-\sigma_{l}^{-}(\tau)}{e^{i \tau \beta_{l}^{-}} \beta_{l}^{-}}\right), \quad c_{l}=-e^{\frac{i \tau}{\varepsilon^{2}}}, \quad d_{l}=2 e^{i \frac{\tau}{2 \varepsilon^{2}}} \cos \left(\tau \frac{\beta_{l}}{2}\right) .
\end{aligned}
$$

We note that $\left|c_{l}\right|,\left|d_{l}\right|,\left|c_{l}^{0}\right| \lesssim 1,\left|d_{l}^{0}\right|,\left|q_{l}\right|,\left|q_{l}^{*}\right| \lesssim \tau^{2}$ and $\left|p_{l}\right|,\left|p_{l}^{*}\right| \lesssim \tau\left(l \in \mathcal{T}_{M}\right)$.

In practice, the above approach is not suitable due to the difficulty of computing the sine transform coefficients in (2.25) through the integrals given in (2.9). Here, we present an efficient implementation by choosing $\psi_{M}^{0}(x)$ as the interpolation of $\psi_{0}(x)$ on the grid points $\left\{x_{j}, j \in \mathcal{T}_{M}\right\}$, i.e., $\psi_{M}(x, 0)=$ $I_{M}\left(\psi_{0}\right)(x)$, and approximating the integrals in (2.9) and (2.25) by a quadrature rule on the grid points.

Now, we state the exponential wave integrator sine pseudospectral (EWI-SP) method. Let $\psi_{j}^{n}$ $(n \geq 1)$ be the approximation of $\psi\left(x_{j}, t_{n}\right)$ with $\psi_{0}^{n}=\psi_{M}^{n}=0$, and we denote $\psi_{j}^{0}=\psi_{0}\left(x_{j}\right), \omega_{j}^{\varepsilon}=\omega^{\varepsilon}\left(x_{j}\right)$ $\left(j \in \mathcal{T}_{M}\right), \psi^{n}=\left(\psi_{0}^{n}, \psi_{1}^{n}, \ldots, \psi_{M}^{n}\right)^{T} \in Y_{M}$. Thus, the numerical approximation $\psi^{n+1} \in Y_{M}$ at time $t_{n+1}$ $(n=0,1, \ldots$,$) can be computed as$

$$
\psi_{j}^{n+1}=\sum_{l=1}^{M-1}\left(\widetilde{\psi^{n+1}}\right)_{l} \sin (j l \pi / M), \quad j \in \mathcal{T}_{M}, \quad n=0,1, \ldots,
$$

where the coefficients are obtained through the following formula,

$$
\begin{aligned}
\left(\widetilde{\psi^{1}}\right)_{l} & =c_{l}^{0}\left(\widetilde{\psi_{0}}\right)_{l}+d_{l}^{0}\left(\widetilde{\psi_{1}^{\varepsilon}}\right)_{l}+p_{l}\left(\widetilde{\left.\mathcal{F}\left(\psi_{0}\right)\right)_{l}}+q_{l}\left(\widetilde{\mathcal{D}\left(\psi_{0}\right)}\right)_{l}, \quad \text { and for } n \geq 1\right. \text { as } \\
\left(\widetilde{\psi^{n+1}}\right)_{l} & =c_{l}^{1}\left(\widetilde{\psi^{n-1}}\right)_{l}+d_{l}^{1}\left(\widetilde{\psi^{n}}\right)_{l}+p_{l}\left(\widetilde{\mathcal{F}\left(\psi^{n}\right)}\right)_{l}+q_{l}\left(\widetilde{\mathcal{D}\left(\psi^{n}\right)}\right)_{l}-p_{l}^{*}\left(\widetilde{\left.\mathcal{F}\left(\psi^{n-1}\right)\right)_{l}}-q_{l}^{*}\left(\widetilde{\left.\mathcal{D}\left(\psi^{n-1}\right)\right)_{l}},\right.\right.
\end{aligned}
$$


and $\mathcal{D}\left(\psi_{0}\right)$ is defined by (2.23) as

$$
\mathcal{D}\left(\psi_{0}\right)=G\left(\psi_{0}\right) \psi_{1}^{\varepsilon}+H\left(\psi_{0}\right) \overline{\psi_{1}^{\varepsilon}}, \quad \mathcal{D}\left(\psi^{n}\right)=\delta_{t}^{-} \mathcal{F}\left(\psi^{n}\right), \quad n \geq 1,
$$

with $c_{l}^{0}, d_{l}^{0}, c_{l}, d_{l}, p_{l}, q_{l}, p_{l}^{*}$ and $q_{l}^{*}$ given in (2.27). In computation, sometimes $\psi_{1}(2.3)$ is not explicitly known, we can replace $\left(\widetilde{\psi_{1}^{\varepsilon}}\right)_{l}\left(l \in \mathcal{T}_{M}\right)$ in $(2.29)$ by the approximation below, and the main results remain the same,

$$
\left(\widetilde{\psi_{1}^{\varepsilon}}\right)_{l} \approx-i\left(\mu_{l}\right)^{2}\left(\widetilde{\psi_{0}}\right)_{l}+i\left(\widetilde{\mathcal{F}\left(\psi_{0}\right)}\right)_{l}+\varepsilon^{\alpha}\left(\widetilde{\omega^{\varepsilon}}\right)_{l} .
$$

The EWI-SP (2.28)-(2.30) is explicit, and can be solved efficiently by fast sine transform. The memory cost is $O(M)$ and the computational cost per time step is $O(M \log M)$. Actually, the scheme has a very good recursion property in phase space (cf. (3.32) and Lemma 3.4). The observation is the following: from the equation (2.1), we can see that there are two characteristics corresponding to the operators $U_{+}(t)=e^{i \frac{1+\sqrt{1+4 \varepsilon^{2}\left(-\nabla^{2}\right)}}{2 \varepsilon^{2}}}$ and $U_{-}(t)=e^{i t \frac{2 \nabla^{2}}{1+\sqrt{1+4 \varepsilon^{2}\left(-\nabla^{2}\right)}}}$. As $\varepsilon \rightarrow 0^{+}$, the first characteristic component $U_{+}$will vanish while oscillating in time, and the second characteristic component $U_{-}$will converge to the Schrödinger operator. That is to say, the solution behaves differently along the two characteristics. In our scheme EWI-SP, these characteristics are treated separately through the approximations of the integrals in (2.18) and (2.19), unlike those wave-type equations (without $i \partial_{t}$ term) [5] and second order ODEs [14, 18].

The pseudospectral method EWI-SP (2.28)-(2.29) is a full discretization and the spectral method (2.24)-(2.25) is a semi-discretization. For simplicity, we will prove the error estimates for the full discretization and omit the analysis for the semi-discretization which can be done in the same spirit.

REMARK 2.1. If we consider the NLSW (2.1) with periodic boundary condition or homogenous Neumann boundary condition, similar Fourier pseudospectral method or cosine pseudospectral method can be easily designed as above, and the main results in this paper remain valid in both cases.

2.2. Main results. In order to state our main results, we introduce the convenient Sobolev spaces. Let $\phi(x) \in H^{m}(\Omega) \cap H_{0}^{1}(\Omega)$ be represented in sine series as

$$
\phi(x)=\sum_{l=1}^{+\infty} \widehat{\phi}_{l} \Phi_{l}(x), \quad x \in \Omega=(a, b),
$$

with $\widehat{\phi}_{l}$ and $\Phi_{l}(x)$ given in (2.9) and (2.6), respectively. Define the subspace of $H^{m} \cap H_{0}^{1}$ as $H_{s}^{m}(\Omega)=$ $\left\{\phi \in H^{m}(\Omega) \mid \partial_{x}^{2 k} \phi(a)=\partial_{x}^{2 k} \phi(b)=0,0 \leq 2 k<m\right\}$ (the boundary values are understood in the trace sense) equipped with the norm

$$
\|\phi\|_{H_{s}^{m}(\Omega)}=\left(\sum_{l=1}^{\infty} \mu_{l}^{2 m}\left|\widehat{\phi}_{l}\right|^{2}\right)^{\frac{1}{2}}
$$

which is equivalent to the $H^{m}$ norm in this subspace. In the remaining part of this paper, we will omit $\Omega$ when the norm is only taken with respect to the spatial variables.

For $u=\left(u_{0}, u_{1}, \ldots, u_{M}\right)^{T} \in Y_{M}$, we define the discrete $l^{2}$, semi- $H^{1}$ and $l^{\infty}$ norms as

$$
\|u\|_{l^{2}}^{2}=h \sum_{j=1}^{M-1}\left|u_{j}\right|^{2}, \quad\left\|\delta_{x}^{+} u\right\|_{l^{2}}^{2}=h \sum_{j=0}^{M-1}\left|\delta_{x}^{+} u_{j}\right|^{2}, \quad\|u\|_{l^{\infty}}=\max _{j \in \mathcal{T}_{M}}\left|u_{j}\right| .
$$


According to the known results in [8,22,25,29] and the asymptotic expansion in section 1, we make the following assumptions on NLSW (2.1), i.e. assumptions on the initial data (2.3) for (2.1),

$$
1 \lesssim\left\|\omega^{\varepsilon}(\cdot)\right\|_{H_{s}^{m_{0}}} \lesssim 1, \quad \psi_{0} \in H_{s}^{m_{0}+2} \quad \text { for some } \quad m_{0} \geq 2
$$

and assumptions on the solution $\psi(t):=\psi(\cdot, t)$ of the NLSW (2.1) and the solution $\psi^{s}(\cdot, t)$ of the NLS (2.2), i.e., let $0<T<T_{\max }$ with $T_{\max }$ being the maximal common existing time,

$$
\|\psi\|_{L^{\infty}\left([0, T] ; L^{\infty} \cap H^{m_{0}} \cap H_{0}^{1}\right)}+\left\|\partial_{t} \psi\right\|_{L^{\infty}\left([0, T] ; H^{1}\right)}+\left\|\psi^{s}\right\|_{L^{\infty}\left([0, T] ; L^{\infty} \cap H^{m_{0}} \cap H_{0}^{1}\right)} \lesssim 1, \quad m_{0} \geq 2,
$$

and $\left\|\partial_{t t} \psi\right\|_{L^{\infty}\left([0, T] ; H^{1}\right)} \lesssim \frac{1}{\varepsilon^{2-\alpha^{*}}}, \quad\left\|\partial_{t}^{k} \psi^{s}\right\|_{L^{\infty}\left([0, T] ; H^{1}\right)} \lesssim 1, \quad\left\|\psi-\psi^{s}\right\|_{L^{\infty}\left([0, T] ; H^{1}\right)} \lesssim \varepsilon^{2}, \quad k=1,2$.

Under assumption (A), we can verify that $\psi_{1}, \psi_{1}^{\varepsilon} \in H_{s}^{m_{0}}(2.3)$ for function $f(\cdot)$ smooth enough. Under assumption (B), in view of the equations (2.1) and (2.2), and Lemma 3.3. it is easy to show that $\psi, \psi^{s} \in L^{\infty}\left([0, T] ; H_{s}^{m_{0}}\right)$.

Denote the constant $M_{1}$ as

$$
M_{1}=\max \left\{\sup _{\varepsilon \in(0,1]}\|\psi(x, t)\|_{L^{\infty}([0, T] \times \Omega)},\left\|\psi^{s}(x, t)\right\|_{L^{\infty}([0, T] \times \Omega)}\right\} .
$$

We can prove the following error estimates for EWI-SP (2.28)-(2.30).

Theorem 2.1. (Well-prepared initial data) Let $\psi^{n} \in Y_{M}$ and $\psi_{I}^{n}(x)=I_{M}\left(\psi^{n}\right)(n \geq 0)$ be the numerical approximations obtained from EWI-SP (2.28)-(2.30). Assume $f(s) \in C^{k}([0,+\infty))(k \geq 3)$, under assumptions (A) and (B), there exist constants $0<\tau_{0}, \frac{h_{0}}{\pi} \leq 1$ independent of $\varepsilon$, if $0<h \leq h_{0}$ and $0<\tau \leq \tau_{0}$, we have for $\alpha \geq 2$, i.e. the well-prepared initial data case,

$$
\begin{aligned}
& \left\|\psi\left(\cdot, t_{n}\right)-\psi_{I}^{n}(\cdot)\right\|_{L^{2}} \lesssim h^{m}+\tau^{2}, \quad\left\|\psi^{n}\right\|_{l \infty} \leq M_{1}+1, \\
& \left\|\nabla\left(\psi\left(\cdot, t_{n}\right)-\psi_{I}^{n}(\cdot)\right)\right\|_{L^{2}} \lesssim h^{m-1}+\tau^{2}, \quad 0 \leq n \leq \frac{T}{\tau},
\end{aligned}
$$

where $m=\min \left\{m_{0}, k\right\}$ and $M_{1}$ is defined in (2.35).

Similarly, we have for the ill-prepared initial data case.

THEOREM 2.2. (Ill-prepared initial data) Under the same condition of Theorem 2.1, there exist constants $0<\tau_{0}, \frac{h_{0}}{\pi} \leq 1$ independent of $\varepsilon$, if $0<h \leq h_{0}$ and $0<\tau \leq \tau_{0}$, we have for $\alpha \in[0,2)$, i.e. the ill-prepared initial data case,

$$
\begin{aligned}
& \left\|\psi\left(\cdot, t_{n}\right)-\psi_{I}^{n}(\cdot)\right\|_{L^{2}} \lesssim h^{m}+\frac{\tau^{2}}{\varepsilon^{2-\alpha}}, \quad\left\|\nabla\left(\psi\left(\cdot, t_{n}\right)-\psi_{I}^{n}(\cdot)\right)\right\|_{L^{2}} \lesssim h^{m-1}+\frac{\tau^{2}}{\varepsilon^{2-\alpha}}, \\
& \left\|\psi\left(\cdot, t_{n}\right)-\psi_{I}^{n}(\cdot)\right\|_{L^{2}} \lesssim h^{m}+\tau^{2}+\varepsilon^{2}, \quad\left\|\nabla\left(\psi\left(\cdot, t_{n}\right)-\psi_{I}^{n}(\cdot)\right)\right\|_{L^{2}} \lesssim h^{m-1}+\tau^{2}+\varepsilon^{2}, \\
& \left\|\psi^{n}\right\|_{l^{\infty}} \leq M_{1}+1, \quad 0 \leq n \leq \frac{T}{\tau},
\end{aligned}
$$

where $m=\min \left\{m_{0}, k\right\}$ and $M_{1}$ is defined in (2.35). Thus, by taking the minimum of $\varepsilon^{2}$ and $\frac{\tau^{2}}{\varepsilon^{2-\alpha}}$ for $0<\varepsilon \leq 1$, we could obtain uniform error bounds as

$$
\left\|\psi\left(\cdot, t_{n}\right)-\psi_{I}^{n}(\cdot)\right\|_{L^{2}} \leq h^{m}+\tau^{4 /(4-\alpha)}, \quad\left\|\nabla\left[\psi\left(\cdot, t_{n}\right)-\psi_{I}^{n}(\cdot)\right]\right\|_{L^{2}} \lesssim h^{m-1}+\tau^{4 /(4-\alpha)}, \quad 0 \leq n \leq \frac{T}{\tau} .
$$

REMARK 2.2. The main results can be extended directly to the system of $N(N \geq 2)$ coupled nonlinear Schrödinger equations with wave operator (NLSW) as

$$
i \partial_{t} \psi_{k}-\varepsilon^{2} \partial_{t t} \psi_{k}(x, t)+\nabla^{2} \psi_{k}(x, t)+f_{k}\left(\left|\psi_{1}\right|^{2}, \ldots,\left|\psi_{N}\right|^{2}\right) \psi_{k}(x, t)=0, \quad k=1, \ldots, N,
$$


where the initial data and boundary conditions for each $\psi_{k}$ are similarly given as (2.1) and (2.3), and the functions $f_{k}:[0, \infty)^{N} \rightarrow \mathbb{R}(k=1, \ldots, N)$ belong to $C^{k}(k \geq 3)$ function class.

Since the exact solution behaves very differently for the well-prepared initial data $(\alpha \geq 2)$ and the ill-prepared initial data $(0 \leq \alpha<2)$ (see (1.4) and [4]), we treat these two cases separately. For the well-prepared initial data, in order to prove Theorem 2.1, we will compare the EWI-SP approximation (2.28)-(2.31) with the $L^{2}$ projection of the exact solution. Following the steps in section 2.1] where we construct the EWI-SP (2.28)-(2.31), it is easy to find that the errors introduced only come from the trigonometric interpolation (spectral accurate in space) and Gautschi type quadrature [14, 18, for the integrals in (2.16) (second order accurate in time). This observation gives Lemma 3.4 for the local truncation error, which is of spectral order in space and second order in time. In addition, because we use a Gautschi type quadrature, the scheme (2.28)-(2.31) posses a very nice recursive property which leads to the nice recursion formula for the error (3.32). These are the key points in our analysis. In the proof, we also use the cutoff technique for the nonlinear term, discrete Sobolev inequality and the energy method.

For the ill-prepared initial data, in order to prove Theorem 2.2 with two different estimates, we adopt a strategy similar to the finite difference method [4] (cf. diagram (4.1)). The idea for establishing the estimates is analogous to that of Theorem 2.1. The same analysis works for 2D and 3D cases, and we discuss this in Remark 4.1

3. Convergence in the well-prepared initial data case. Before the analysis of convergence, we first review and introduce some lemmas for the projection operator $P_{M}$ and interpolation operator $I_{M}[20,26]$.

Lemma 3.1. ( $L^{2}$ projection) Let $\phi(x) \in H_{s}^{m}(\Omega)(m \geq 2)$, then

$$
\left\|\phi(\cdot)-P_{M}(\phi)(\cdot)\right\|_{L^{2}} \lesssim \pi^{-m} h^{m}, \quad\left\|\nabla\left[\phi(\cdot)-P_{M}(\phi)(\cdot)\right]\right\|_{L^{2}} \lesssim \pi^{1-m} h^{m-1} .
$$

For the sine trigonometric interpolation $I_{M}$, we have similar results.

Lemma 3.2. (Sine interpolation) Let $\phi(x) \in H_{0}^{1}(\Omega)$ and $\phi=\left(\phi_{0}, \phi_{1}, \ldots, \phi_{M}\right)^{T}$ with $\phi_{j}=\phi\left(x_{j}\right)$ $\left(j \in \mathcal{T}_{M}^{0}\right)$, we have

$$
\left\|I_{M}(\phi)(\cdot)\right\|_{L^{2}} \leq\|\phi(\cdot)\|_{L^{2}}+h\|\nabla \phi(\cdot)\|_{L^{2}}, \quad\left\|\delta_{x}^{+} \phi\right\|_{l^{2}} \leq\left\|\nabla I_{M}(\phi)(\cdot)\right\|_{L^{2}} \leq \frac{\pi}{2}\left\|\delta_{x}^{+} \phi\right\|_{l^{2}} .
$$

$I_{M}$ and $P_{M}$ are identity transforms on $X_{M}$, and if $\phi(x) \in X_{M}$,

$$
\|\nabla \phi(\cdot)\|_{L^{2}} \lesssim h^{-1}\|\phi(\cdot)\|_{L^{2}}
$$

Proof. The lemma can be proved analogous to [20,26. We prove the first statement here for reader's convenience. By definition of $I_{M}$, using Cauchy inequality, it is easy to check that

$$
\begin{aligned}
\left\|I_{M}(\phi)(\cdot)\right\|_{L^{2}}^{2} & =h \sum_{j=1}^{M-1}\left|\phi\left(x_{j}\right)\right|^{2}=\|\phi(\cdot)\|_{L^{2}}^{2}-\sum_{j=0}^{M-1} \int_{x_{j}}^{x_{j+1}}\left(|\phi(\mathbf{x})|^{2}-\left|\phi\left(x_{j}\right)\right|^{2}\right) d \mathbf{x} \\
& =\|\phi(\cdot)\|_{L^{2}}^{2}-\sum_{j=0}^{M-1} \int_{x_{j}}^{x_{j+1}} \int_{x_{j}}^{\mathbf{x}} \partial_{x}\left|\phi\left(\mathbf{x}^{\prime}\right)\right|^{2} d \mathbf{x}^{\prime} d \mathbf{x} \\
& \leq\|\phi(\cdot)\|_{L^{2}}^{2}+2 h\|\nabla \phi(\cdot)\|_{L^{2}}\|\phi(\cdot)\|_{L^{2}},
\end{aligned}
$$


which implies the first conclusion by using Cauchy inequality. For the second conclusion, we note that $\left\|\nabla I_{M}(\phi)(\cdot)\right\|_{2}^{2}=\frac{b-a}{2} \sum_{l=1}^{M-1}\left|\mu_{l}\right|^{2}\left|\widetilde{\phi}_{l}\right|^{2}, \quad \delta_{x}^{+} \phi\left(x_{j}\right)=\sum_{l=0}^{M-1} \frac{2}{h} \widetilde{\phi}_{l} \sin \left(\frac{\mu_{l} h}{2}\right) \cos \left(\mu_{l}\left(j+\frac{1}{2}\right) h\right), \quad 0 \leq j \leq M-1$,

thus by Parseval's identity, we know

$$
\left\|\delta_{x}^{+} \phi\right\|_{l^{2}}^{2}=\frac{h M}{2} \sum_{l=0}^{M-1}\left|\mu_{l}\right|^{2}\left|\widetilde{\phi}_{l}\right|^{2}\left|\operatorname{sinc}\left(\frac{\mu_{l} h}{2}\right)\right|^{2} .
$$

Recalling that $0<\frac{1}{2} \mu_{l} h \leq \frac{\pi}{2}\left(l \in \mathcal{T}_{M}\right)$, sinc $\left(\frac{\mu_{l} h}{2}\right) \in\left[\frac{2}{\pi}, 1\right]$, we get the second conclusion.

For $I_{M}$ applied to the nonlinear term, we require some regularity to achieve the desired accuracy.

Lemma 3.3. For $\phi(x) \in H_{s}^{m}(\Omega)(m \geq 2)$ and $f(\cdot) \in C^{k}([0, \infty))(k \geq 2)$, we have

$$
\mathcal{F}(\phi)=f\left(|\phi|^{2}\right) \phi \in H_{s}^{m^{*}}, \quad \text { with } \quad\|\mathcal{F}(\phi)\|_{H_{s}^{m^{*}}} \lesssim\|\phi\|_{H_{s}^{m^{*}}}, \quad m^{*}=\min \{m, k\} .
$$

Proof. This is essentially due to the fact that $\mathcal{F}(\phi)$ maps an odd function to an odd function. The results can be checked by direct computation and we omit it here. $\square$

Using Lemmas 3.1 and 3.2 we find for any $\Phi \in H_{0}^{1}$,

$$
\left\|I_{M}(\Phi)-P_{M}(\Phi)\right\|_{L^{2}}=\left\|I_{M}\left[\Phi-P_{M}(\Phi)\right]\right\|_{L^{2}} \lesssim\left\|\Phi-P_{M}(\Phi)\right\|_{L^{2}}+h\left\|\nabla\left(\Phi-P_{M}(\Phi)\right)\right\|_{L^{2}} .
$$

Let $\psi(x, t)$ be the solution of the NLSW (2.1), we write $\psi(t)$ in short of $\psi(x, t)$. In order to prove the main theorem, we define the 'local truncation error' $\xi^{n}(x)=\sum_{l=1}^{M-1}\left(\widetilde{\xi^{n}}\right)_{l} \Phi_{l}(x) \in X_{M}$ for $l \in \mathcal{T}_{M}$ as

$$
\begin{aligned}
\left(\widetilde{\left.\xi^{0}\right)_{l}}=\right. & c_{l}^{0}\left(\widehat{\psi_{0}}\right)_{l}+d_{l}^{0}\left(\widehat{\psi_{1}^{\varepsilon}}\right)_{l}+p_{l}\left(\widetilde{\mathcal{F}\left(\psi_{0}\right)}\right)_{l}+q_{l}\left(\widetilde{\mathcal{D}\left(\psi_{0}\right)}\right)_{l}-(\widehat{\psi(\tau)})_{l}, \\
\left(\widetilde{\xi^{n}}\right)_{l}= & \left.\left.\left.c_{l}\left(\widehat{\left(t_{n-1}\right.}\right)\right)_{l}+d_{l}\left(\widehat{\psi\left(t_{n}\right)}\right)_{l}+p_{l}\left(\widetilde{\mathcal{F}\left(\psi\left(t_{n}\right)\right.}\right)\right)_{l}+q_{l}\left(\widetilde{\mathcal{D}\left(\psi\left(t_{n}\right)\right.}\right)\right)_{l}-p_{l}^{*}\left(\widetilde{\mathcal{F}\left(\psi_{0}\right)}\right)_{l} \\
& -q_{l}^{*}\left(\mathcal{D}\left(\widetilde{\psi\left(t_{n-1}\right)}\right)\right)-\left(\widetilde{\psi\left(t_{n+1}\right)}\right)_{l}, \quad n \geq 1 .
\end{aligned}
$$

Then we have the following results.

Lemma 3.4. Let $\xi^{n}(x)(n \geq 0)$ be defined as 3.7), $f(\cdot) \in C^{k}([0, \infty))(k \geq 3)$, under assumptions (A) and (B), we have the following decomposition of $\xi^{n}(x)(n \geq 0)$ (3.12),

$$
\begin{aligned}
& \xi^{0}(x)=e^{i \tau \beta_{l}^{+}} \xi^{0,+}(x)-e^{i \tau \beta_{l}^{-}} \xi^{0,-}(x), \\
& \xi^{n}(x)=e^{i \tau \beta_{l}^{+}} \xi^{n,+}(x)-e^{i \tau \beta_{l}^{-}} \xi^{n,-}(x)-e^{\frac{i \tau}{\varepsilon^{2}}}\left(\xi^{n-1,+}(x)-\xi^{n-1,-}(x)\right), \quad n \geq 1,
\end{aligned}
$$

where $\left.\xi^{n, \pm}(x)=\sum_{l=1}^{M-1} \widetilde{\left(\xi^{n, \pm}\right.}\right)_{l} \Phi_{l}(x) \in X_{M}$ and for $\alpha \geq 2$, i.e., the well-prepared initial data case, we have

$$
\left\|\xi^{n, \pm}(\cdot)\right\|_{L^{2}} \lesssim \tau h^{m}+\tau^{3}, \quad\left\|\nabla \xi^{n, \pm}(\cdot)\right\|_{L^{2}} \lesssim \tau h^{m-1}+\tau^{3}, \quad m=\min \left\{m_{0}, k\right\}, \quad n \geq 0 ;
$$

for $\alpha \in[0,2)$, i.e. the ill-prepared initial data case, we have

$$
\left\|\xi^{n, \pm}(\cdot)\right\|_{L^{2}} \lesssim \tau h^{m}+\frac{\tau^{3}}{\varepsilon^{2-\alpha}}, \quad\left\|\nabla \xi^{n, \pm}(\cdot)\right\|_{L^{2}} \lesssim \tau h^{m-1}+\frac{\tau^{3}}{\varepsilon^{2-\alpha}}, \quad m=\min \left\{m_{0}, k\right\}, \quad n \geq 0 .
$$


In addition, the coefficients $p_{l}, q_{l}, p_{l}^{*}$ and $q_{l}^{*}(n \geq 0)$ given in 2.27) can be split as

$$
\begin{aligned}
& p_{l}=e^{i \tau \beta_{l}^{+}} p_{l}^{+}-e^{i \tau \beta_{l}^{-}} p_{l}^{-}, \quad q_{l}=e^{i \tau \beta_{l}^{+}} q_{l}^{+}-e^{i \tau \beta_{l}^{-}} q_{l}^{-}, \quad p_{l}^{*}=e^{\frac{i \tau}{\varepsilon^{2}}}\left(p_{l}^{+}-p_{l}^{-}\right), \quad q_{l}^{*}=e^{\frac{i \tau}{\varepsilon^{2}}}\left(q_{l}^{+}-q_{l}^{-}\right), \\
& p_{l}^{+}=\frac{-i \tau}{\varepsilon^{2} \beta_{l}} \overline{\sigma_{l}^{+}(\tau)}, \quad p_{l}^{-}=\frac{-i \tau}{\varepsilon^{2} \beta_{l}} \overline{\sigma_{l}^{-}(\tau)}, \quad q_{l}^{+}=\frac{\tau}{\varepsilon^{2} \beta_{l}} \cdot \frac{1-\sigma_{l}^{+}(\tau)}{e^{i \tau \beta_{l}^{+} \beta_{l}^{+}}}, \quad q_{l}^{-}=\frac{\tau}{\varepsilon^{2} \beta_{l}} \cdot \frac{1-\sigma_{l}^{-}(\tau)}{e^{i \tau \beta_{l}^{-}} \beta_{l}^{-}},
\end{aligned}
$$

where $\left|p_{l}^{ \pm}\right| \lesssim \tau,\left|q_{l}^{ \pm}\right| \lesssim \tau^{2}$

Proof. Multiplying both sides of NLSW (2.1) by $\Phi_{l}(x)=\sin \left(\mu_{l}(x-a)\right)$ and integrating over $\Omega$, we easily recover the equations for $(\widehat{\psi})_{l}(t)$, which are exactly the same as (2.12) with $\psi_{M}$ being replaced by $\psi(x, t)$. Replacing $\psi_{M}$ with $\psi(x, t)$, we use the same notations $f_{l}^{n}(s)$ as in (2.14), and it is worth noticing that the time derivatives of $f_{l}^{n}(s)$, enjoy the same properties of time derivatives for $\mathcal{F}(\psi(x, t))$. Thus, the same representations (2.18) and (2.19) hold for $\left(\overline{\psi\left(t_{n}\right)}\right)_{l}$ with $n=1$ and $n \geq 2$, respectively.

Now it is clear that the error $\xi^{n}(x)$ comes from the approximations for the integrals (2.25) and trigonometric interpolation (2.29). First, we can write the integrals in (2.18) and (2.19) as

$$
\frac{i}{\varepsilon^{2} \beta_{l}} \int_{0}^{\tau} f_{l}^{n}(s) \kappa_{l}(\tau-s) d s=e^{i \tau \beta_{l}^{+}} \frac{i}{\varepsilon^{2} \beta_{l}} \int_{0}^{\tau} f_{l}^{n}(s) e^{-i \beta_{l}^{+} s} d s-e^{i \tau \beta_{l}^{-}} \frac{i}{\varepsilon^{2} \beta_{l}} \int_{0}^{\tau} f_{l}^{n}(s) e^{-i \beta_{l}^{-} s} d s,
$$

then $\xi^{n}(x)(n \geq 0)$ can be written as (3.8) with

$$
\xi^{n,+}(x)=\eta^{n,+}(x)+\zeta^{n,+}(x), \quad \xi^{n,-}(x)=\eta^{n,-}(x)+\zeta^{n,-}(x),
$$

where the interpolation error $\zeta^{n,+}(x), \zeta^{n,-}(x) \in X_{M}(n \geq 0)$ are determined by their sine transform coefficients for $l \in \mathcal{T}_{M}$ given by

$$
\begin{aligned}
& \left(\widehat{\zeta^{0, \pm}}\right)_{l}=p_{l}^{ \pm}\left[\left(\widetilde{\mathcal{F}\left(\psi_{0}\right)}\right)_{l}-\left(\widehat{\mathcal{F}\left(\psi_{0}\right)}\right)_{l}\right]+q_{l}^{ \pm}\left[\left(\widetilde{G\left(\psi_{0}\right) \psi_{1}^{\varepsilon}}\right)_{l}+\left(\widehat{\left(H\left(\psi_{0}\right) \bar{\psi}_{1}^{\varepsilon}\right.}\right)_{l}-\left(\widehat{G\left(\psi_{0}\right) \psi_{1}^{\varepsilon}}\right)_{l}-\left(\widehat{H\left(\psi_{0}\right) \bar{\psi}_{1}^{\varepsilon}}\right)_{l}\right], \\
& \left.\left.\left.\left.\left(\widehat{\zeta^{n, \pm}}\right)_{l}=p_{l}^{ \pm}\left[\left(\widehat{\mathcal{F}\left(\psi\left(t_{n}\right)\right.}\right)\right)_{l}-\left(\widehat{\mathcal{F}\left(\psi\left(t_{n}\right)\right.}\right)\right)_{l}\right]+q_{l}^{ \pm}\left[\left(\delta_{t}^{-} \widehat{\mathcal{F}\left(\psi\left(t_{n}\right)\right.}\right)\right)_{l}-\left(\delta_{t}^{-} \widehat{\mathcal{F}\left(\psi\left(t_{n}\right)\right.}\right)\right)_{l}\right], \quad n \geq 1,
\end{aligned}
$$

with coefficients $p_{l}^{ \pm}$and $q_{l}^{ \pm}$given in the lemma, and the integral approximation error $\eta^{n,+}(x), \eta^{n,-}(x) \in$ $X_{M}(n \geq 0)$ are given by their sine transform coefficients as, for $l \in \mathcal{T}_{M}$,

$$
\begin{aligned}
& \left(\widehat{\left.\eta^{0, \pm}\right)_{l}}=-\frac{i}{\varepsilon^{2} \beta_{l}} \int_{0}^{\tau} \int_{0}^{s} \int_{0}^{s_{1}}\left(\partial_{t t} f_{l}^{0}\left(s_{2}\right)\right) e^{-i s \beta_{l}^{ \pm}} d s_{2} d s_{1} d s, \quad \text { and for } n \geq 1,\right. \\
& \left(\widehat{\eta^{n, \pm}}\right)_{l}=-\frac{i}{\varepsilon^{2} \beta_{l}} \int_{0}^{\tau}\left(\int_{0}^{s} \int_{0}^{s_{1}} \partial_{t t} f_{l}^{n}\left(s_{2}\right) d s_{2} d s_{1}-s \tau \int_{0}^{1} \int_{\theta}^{1} \partial_{t t} f_{l}^{n-1}\left(\theta_{1} \tau\right) d \theta_{1} d \theta\right) e^{-i s \beta_{l}^{ \pm}} d s .
\end{aligned}
$$

Now, to obtain estimates (3.9) and (3.10), we only need to estimate $\zeta^{n, \pm}$ and $\eta^{n, \pm}$.

First, let us consider $\zeta^{n, \pm}$. For $n=0$, recalling assumptions (A) and (B), it is easy to verify that $\psi_{0}, \psi(\tau)$ belong to $H_{s}^{m_{0}}$ and $\psi_{1}^{\varepsilon} \in H_{s}^{m}\left(m=\min \left\{m_{0}, k\right\}\right)$, while $\mathcal{F}\left(\psi_{0}\right)$ belongs to $H_{s}^{\min \left\{m_{0}, k\right\}}, G\left(\psi_{0}\right) \psi_{1}^{\varepsilon}$ and $H\left(\psi_{0}\right) \overline{\psi_{1}^{\varepsilon}}$ belong to $H_{s}^{m^{*}}\left(m^{*}=\min \{m, k-1\} \geq 2\right)$. Hence, noticing (3.6) and Lemma 3.1] we know

$$
\left\|\zeta^{0, \pm}(\cdot)\right\|_{L^{2}} \lesssim \tau\left(h^{m}+\tau h^{m^{*}}\right) \lesssim \tau\left(h^{m}+\tau^{2}\right), \quad\left\|\nabla \zeta^{0, \pm}(\cdot)\right\|_{L^{2}} \lesssim \tau\left(h^{m-1}+\tau^{2}\right)
$$

Similarly, we can get for $n \geq 1$ that

$$
\left\|\zeta^{n, \pm}(\cdot)\right\|_{L^{2}} \lesssim \tau h^{m}, \quad\left\|\nabla \zeta^{n, \pm}(\cdot)\right\|_{L^{2}} \lesssim \tau h^{m-1} .
$$


Then we estimate $\eta^{n, \pm}$. Noticing $f \in C^{3}$ and assumptions (A) and (B), in view of the particular assumption $\partial_{t t} \psi \sim \varepsilon^{\alpha^{*}-2}$, we can deduce that for $t \in[0, T]$,

$$
\left\|\partial_{t t} \mathcal{F}(\psi(t))\right\|_{L^{2}} \lesssim \varepsilon^{\alpha^{*}-2}, \quad\left\|\nabla\left[\partial_{t t} \mathcal{F}(\psi(t))\right]\right\|_{L^{2}} \lesssim \varepsilon^{\alpha^{*}-2} .
$$

The key point is the behavior of $\partial_{t t} \psi$. Substituting all the above results into (3.14), we can easily get

$$
\left.\left|\left(\widehat{\eta^{0}}\right)_{l}\right| \lesssim \int_{0}^{\tau} \int_{0}^{s} \int_{0}^{s_{1}}\left(\mid\left(\partial_{t t} \widehat{\mathcal{F}\left(\psi\left(s_{2}\right)\right.}\right)\right)_{l} \mid\right) d s_{2} d s_{1} d s, \quad l \in \mathcal{T}_{M}
$$

and by applying Cauchy inequalities and Bessel inequalities as well as (3.17), we obtain

$$
\begin{aligned}
\left\|\eta^{0, \pm}(\cdot)\right\|_{L^{2}}^{2} & =\frac{b-a}{2} \sum_{l=1}^{M-1}\left|\left(\widehat{\eta^{0}}\right)_{l}\right|^{2} \lesssim \frac{(b-a) \tau^{3}}{2} \int_{0}^{\tau} \int_{0}^{s} \int_{0}^{s_{1}}\left(\sum_{l=1}^{M-1}\left|\left(\partial_{t t} \widehat{\mathcal{F}\left(\psi\left(s_{2}\right)\right)}\right)_{l}\right|^{2}\right) d s_{2} d s_{1} d s \\
& \lesssim \tau^{3} \int_{0}^{\tau} \int_{0}^{s} \int_{0}^{s_{1}}\left(\left\|\partial_{t t} \mathcal{F}\left(\psi\left(s_{2}\right)\right)\right\|_{L^{2}}^{2}\right) d s_{2} d s_{1} d s \lesssim \tau^{6} \varepsilon^{2 \alpha^{*}-4} .
\end{aligned}
$$

In the same spirit, we can obtain

$$
\left\|\nabla \eta^{0, \pm}(\cdot)\right\|_{L^{2}}^{2}=\frac{b-a}{2} \sum_{l=1}^{M-1}\left|\mu_{l}\right|^{2}\left|\left(\widehat{\eta^{0}}\right)_{l}\right|^{2} \lesssim \tau^{3} \int_{0}^{\tau} \int_{0}^{s} \int_{0}^{s_{1}}\left(\left\|\nabla \partial_{t t} \mathcal{F}\left(\psi\left(s_{2}\right)\right)\right\|_{L^{2}}^{2}\right) d s_{2} d s_{1} d s \lesssim \tau^{6} \varepsilon^{2 \alpha^{*}-4}
$$

Thus, we have proved that $\left\|\eta^{0, \pm}(\cdot)\right\|_{L^{2}}+\left\|\nabla \eta^{0, \pm}(\cdot)\right\|_{L^{2}} \lesssim \tau^{3} \varepsilon^{2-\alpha^{*}}$. Using the same approach (omitted here for brevity), we can deduce that

$$
\left\|\eta^{n, \pm}(\cdot)\right\|_{L^{2}}+\left\|\nabla \eta^{n, \pm}(\cdot)\right\|_{L^{2}} \lesssim \tau^{3} \varepsilon^{\alpha^{*}-2}, \quad \text { for } \quad n \geq 1
$$

Now, we are ready to prove the lemma. By combining all the results above and triangle inequality, we finally prove (3.9) and (3.10) if we separate the cases $\alpha \geq 2$ and $\alpha \in[0,2)$.

Now, let us look back at our main results. Making use of explicit property of the proposed scheme, we know that if the error estimates are correct, we can replace the nonlinearity $\mathcal{F}(z)=f\left(|z|^{2}\right) z$ by a cut-off nonlinearity $\mathcal{F}_{B}(z)$ defined as

$$
\mathcal{F}_{B}(z)=\rho\left(\frac{|z|^{2}}{B}\right) f\left(|z|^{2}\right) z, \quad \text { where } \quad \rho(s) \in C_{0}^{\infty}(\mathbb{R}) \quad \text { satisfying } \quad \rho(s)= \begin{cases}1, & |s| \leq 1 \\ 0, & |s| \geq 2 \\ \in[0,1], & |s| \in[1,2]\end{cases}
$$

where $B=\left(M_{1}+1\right)^{2}$ with $M_{1}$ given in (2.35). It is true that this replacement doesn't affect NLSW and NLS themselves. Thus, if the same error estimates in Theorem 2.1 and 2.2 hold for NLSW with the truncated nonlinearity $\mathcal{F}_{B}(z)$, it is a direct consequence that the error estimates (Theorem 2.1 and 2.2) hold true for NLSW with nonlinearity $\mathcal{F}(z)$ as the corresponding numerical schemes coincide in this case (see more discussions in [2, 4]).

Based on the above observation, we only need to prove Theorems 2.1 and 2.2 for the nonlinearity $\mathcal{F}(z)$ replaced by $\mathcal{F}_{B}(z)$. From now on, we will treat $\mathcal{F}(z)$ as $\mathcal{F}_{B}(z)$ while not changing the notation.

Proof of Theorem 2.1. Let us define the error $e^{n} \in Y_{M}$ and $e^{n}(x) \in X_{M}(n \geq 0)$ as

$$
\begin{aligned}
& e_{j}^{n}=\left(P_{M} \psi\left(t_{n}\right)\right)\left(x_{j}\right)-\psi_{j}^{n}, \quad j \in \mathcal{T}_{M}^{0}, \quad n \geq 0 \\
& e^{n}(x)=I_{M}\left(e^{n}\right)(x)=\sum_{l=1}^{M-1}\left(\widetilde{e^{n}}\right)_{l} \Phi_{l}(x), \quad n \geq 0, \quad x \in \Omega .
\end{aligned}
$$


Then, by triangle inequality and Lemma 3.2 as well as assumptions (A) and (B), we obtain

$$
\begin{aligned}
& \left\|\psi\left(\cdot, t_{n}\right)-\psi_{I}^{n}(\cdot)\right\|_{L^{2}} \leq\left\|\psi\left(\cdot, t_{n}\right)-P_{M}\left(\psi\left(t_{n}\right)\right)(\cdot)\right\|_{L^{2}}+\left\|e^{n}(\cdot)\right\|_{L^{2}} \lesssim h^{m_{0}}+\left\|e^{n}(\cdot)\right\|_{L^{2}}, \\
& \left\|\nabla\left[\psi\left(\cdot, t_{n}\right)-\psi_{I}^{n}(\cdot)\right]\right\|_{L^{2}} \lesssim h^{m_{0}-1}+\left\|\nabla e^{n}(\cdot)\right\|_{L^{2}}, \quad n \geq 0 .
\end{aligned}
$$

Applying inverse inequality, and Lemma 3.2 further, we have

$$
\begin{aligned}
\left\|\psi^{n}\right\|_{l^{\infty}} & \leq \sup _{j \in \mathcal{T}_{M}^{0}}\left|\psi\left(x_{j}, t_{n}\right)-\psi_{j}^{n}\right|+\left\|\psi\left(t_{n}\right)\right\|_{L^{\infty}} \leq \sup _{j \in \mathcal{T}_{M}^{0}}\left|e_{j}^{n}+\psi\left(x_{j}, t_{n}\right)-P_{M}\left(\psi\left(t_{n}\right)\right)\left(x_{j}\right)\right|+M_{1} \\
& \leq M_{1}+\left\|e^{n}\right\|_{l^{\infty}}+\sup _{j \in \mathcal{T}_{M}^{0}}\left|I_{M}\left(\psi\left(t_{n}\right)\right)\left(x_{j}\right)-P_{M}\left(\psi\left(t_{n}\right)\right)\left(x_{j}\right)\right| \\
& \leq M_{1}+\left\|e^{n}\right\|_{l^{\infty}}+C_{1} h^{-d / 2}\left\|I_{M}\left(\psi\left(t_{n}\right)\right)(\cdot)-P_{M}\left(\psi\left(t_{n}\right)\right)(\cdot)\right\|_{L^{2}} \\
& \leq M_{1}+C_{2} h^{m_{0}-d / 2}+\left\|e^{n}\right\|_{l^{\infty}} \leq M_{1}+\frac{1}{2}+\left\|e^{n}\right\|_{l^{\infty}}, \quad n \geq 0,
\end{aligned}
$$

where $C_{1}$ and $C_{2}$ depend on $\|\psi\|_{L^{\infty}\left([0, T] ; H_{s}^{m_{0}}\right)}$ and $0<h<h_{1}$ for some $h_{1}>0, d$ is the dimension of the spatial space, i.e., $d=1$ in the current case. However, we put $d$ here to indicate how the proof works for two and three dimensions $(d=2,3)$ (see also Remark 4.1). As a consequence of the discrete Sobolev inequality in 1D,

$$
\left\|e^{n}\right\|_{l^{\infty}}^{2} \lesssim\left\|e^{n}\right\|_{l^{2}}\left\|\delta_{x}^{+} e^{n}\right\|_{l^{2}} \lesssim\left\|e^{n}(\cdot)\right\|_{L^{2}}\left\|\nabla e^{n}(\cdot)\right\|_{L^{2}}
$$

we only need to estimate the $L^{2}$ and semi- $H^{1}$ norms of $e^{n}(x)(n \geq 0)$.

For $e^{0}$ and $e^{1}$. Considering $e^{0}(x)$, we note

$$
e^{0}(x)=P_{M}\left(\psi_{0}\right)(x)-I_{M}\left(\psi_{0}\right)(x)
$$

in view of Lemma 3.2 and (3.6), we get $\left\|e^{0}(\cdot)\right\|_{L^{2}} \lesssim h^{m_{0}} \lesssim h^{m}$ and $\left\|\nabla e^{0}(\cdot)\right\|_{L^{2}} \lesssim h^{m_{0}-1} \lesssim h^{m-1}$, where $m=\min \left\{m_{0}, k\right\}$. For $e^{1}(x)$, we have $\psi_{1}^{\varepsilon} \in H_{s}^{m}$ and

$$
\left(\widetilde{e^{1}}\right)_{l}=-\left(\widetilde{\xi^{0}}\right)_{l}+c_{l}^{0}\left(\left(\widehat{\psi_{0}}\right)_{l}-\left(\widetilde{\psi_{0}}\right)_{l}\right)+d_{l}^{0}\left(\left(\widehat{\psi_{1}^{\varepsilon}}\right)_{l}-\left(\widetilde{\psi_{1}^{\varepsilon}}\right)_{l}\right) .
$$

Then Lemma 3.4 implies $\left\|e^{1}(\cdot)\right\|_{L^{2}} \lesssim h^{m}+\tau^{3}$ and $\left\|\nabla e^{1}(\cdot)\right\|_{L^{2}} \lesssim h^{m-1}+\tau^{3}$. It is obvious $\left\|\psi^{0}\right\|_{l^{\infty}} \leq$ $\left\|\psi_{0}\right\|_{L^{\infty}} \leq M_{1}+1$, and by discrete Sobolev inequality, we obtain

$$
\left\|e^{1}\right\|_{l^{\infty}}^{2} \lesssim\left\|e^{1}(\cdot)\right\|_{L^{2}}\left\|\nabla e^{1}(\cdot)\right\|_{L^{2}} \lesssim h^{2 m-2}+\tau^{6},
$$

which implies there exist $h_{2}, \tau_{1}>0$ such that $\left\|e_{j}^{1}\right\|_{l^{\infty}} \leq \frac{1}{2}$, and $\left\|\psi^{1}\right\|_{l^{\infty}} \leq M_{1}+1$ for $0<\tau \leq \tau_{1}$, $0<h \leq h_{2}$ in view of (3.22). This proves the conclusion for $n=0,1$ in Theorem 2.1 by noticing (3.21). Before going to the next step, we note the decomposition as

$$
\left(\widetilde{e^{0}}\right)_{l}=\left(\widetilde{e^{0,+}}\right)_{l}+\left(\widetilde{e^{0,-}}\right)_{l}, \quad\left(\widetilde{e^{1}}\right)_{l}=\left(\widetilde{e^{0,+}}\right)_{l} e^{i \tau \beta_{l}^{+}}+\left(\widetilde{e^{0,-}}\right)_{l} e^{i \tau \beta_{l}^{-}}-\left(\widetilde{\xi^{0}}\right)_{l}, \quad l \in \mathcal{T}_{M},
$$

where $e^{0, \pm}(x)=\sum_{l=1}^{M-1}\left(\widetilde{e^{0, \pm}}\right)_{l} \Phi_{l}(x) \in X_{M}$ are given by

$$
\left(\widetilde{e^{0,+}}\right)_{l}=-\frac{\beta_{l}^{-}}{\beta_{l}}\left[\left(\widehat{\psi_{0}}\right)_{l}-\left(\widetilde{\psi_{0}}\right)_{l}\right]-\frac{i}{\beta_{l}}\left[\left(\widehat{\psi_{1}^{\varepsilon}}\right)_{l}-\left(\widetilde{\psi_{1}^{\varepsilon}}\right)_{l}\right], \quad\left(\widetilde{e^{0,-}}\right)_{l}=\frac{\beta_{l}^{+}}{\beta_{l}}\left[\left(\widehat{\psi_{0}}\right)_{l}-\left(\widetilde{\psi_{0}}\right)_{l}\right]+\frac{i}{\beta_{l}}\left[\left(\widehat{\psi_{1}^{\varepsilon}}\right)_{l}-\left(\widetilde{\psi_{1}^{\varepsilon}}\right)_{l}\right]
$$


We can easily derive that

$$
\left\|e^{0, \pm}(\cdot)\right\|_{L^{2}} \lesssim h^{m}, \quad \text { and } \quad\left\|\nabla e^{0, \pm}(\cdot)\right\|_{L^{2}} \lesssim h^{m-1}
$$

Error equation for $e^{n}(x)(n \geq 2)$. For other time steps, subtracting (3.7) from (2.29) and noticing $e^{i \frac{\tau}{\varepsilon^{2}}}=e^{i \tau \beta_{l}^{+}} \cdot e^{i \tau \beta_{l}^{-}}$and $2 e^{\frac{i \tau}{2 \varepsilon^{2}}} \cos \left(\frac{\tau \beta_{l}}{2}\right)=e^{i \tau \beta_{l}^{+}}+e^{i \tau \beta_{l}^{-}}$, we obtain

$$
\left.\left.\widetilde{\left(e^{n+1}\right.}\right)_{l}=-e^{i \tau\left(\beta_{l}^{+}+\beta_{l}^{-}\right)} \widetilde{\left(e^{n-1}\right.}\right)_{l}+\left(e^{i \tau \beta_{l}^{+}}+e^{i \tau \beta_{l}^{-}}\right)\left(\widetilde{e^{n}}\right)_{l}-\left(\widetilde{\xi^{n}}\right)_{l}+\widetilde{W}_{l}^{n}, \quad n \geq 1, \quad l \in \mathcal{T}_{M},
$$

where $\widetilde{W}_{l}^{n}$ can be written as follows (similar as Lemma 3.4 ),

$$
\begin{aligned}
\widetilde{W}_{l}^{n} & =e^{i \tau \beta_{l}^{+}} \widetilde{W}_{l}^{n,+}-e^{i \tau \beta_{l}^{-}} \widetilde{W}_{l}^{n,-}-e^{\frac{i \tau}{\varepsilon^{2}}}\left(\widetilde{W}_{l}^{n-1,+}-\widetilde{W}_{l}^{n-1,-}\right), \quad n \geq 1, \quad \text { with } \quad \widetilde{W}_{l}^{0, \pm}=0, \\
\widetilde{W}_{l}^{n, \pm} & \left.\left.=p_{l}^{ \pm}\left(\left(\widetilde{\mathcal{F}\left(\psi\left(t_{n}\right)\right.}\right)\right)_{l}-\left(\widetilde{\mathcal{F}\left(\psi^{n}\right)}\right)_{l}\right)+q_{l}^{ \pm}\left(\delta_{t}^{-}\left(\widetilde{\mathcal{F}\left(\psi\left(t_{n}\right)\right.}\right)\right)_{l}-\delta_{t}^{-}\left(\widetilde{\mathcal{F}\left(\psi^{n}\right)}\right)_{l}\right), \quad n \geq 1, l \in \mathcal{T}_{M} .
\end{aligned}
$$

Property of $\widetilde{W}_{l}^{n, \pm}$. Next, we claim that if $\left\|\psi^{n}\right\|_{l^{\infty}} \leq M_{1}+1$, under assumptions (A) and (B), $f \in$ $C^{3}([0, \infty))$ and $\mathcal{F}(z)$ enjoys the properties of $\mathcal{F}_{B}(z)(3.19)$, then we have (see detailed proof in Appendix)

$$
\begin{aligned}
& \frac{b-a}{2} \sum_{l=1}^{M-1}\left|\widetilde{W}_{l}^{n, \pm}\right|^{2} \lesssim \tau^{2} \sum_{k=n-1}^{n}\left\|e^{k}(\cdot)\right\|_{L^{2}}^{2}+\tau^{2} h^{2 m_{0}}, \\
& \frac{b-a}{2} \sum_{l=1}^{M-1} \mu_{l}^{2}\left|\widetilde{W}^{n, \pm}\right|^{2} \lesssim \tau^{2} \sum_{k=n-1}^{n}\left(\left\|e^{k}(\cdot)\right\|_{L^{2}}^{2}+\left\|\nabla\left[e^{k}(\cdot)\right]\right\|_{L^{2}}^{2}\right)+\tau^{2} h^{2 m_{0}-2}, \quad n \geq 1 .
\end{aligned}
$$

Proof by energy method. Using (3.29) iteratively, noticing Lemma 3.4 and above decomposition (3.30), we can find that for $n \geq 1$,

$$
\begin{aligned}
\left.\widetilde{\left(e^{n+1}\right.}\right)_{l}= & \left.\left(\widetilde{e^{0,+}}\right)_{l} e^{i(n+1) \tau \beta_{l}^{+}}+\left(\widetilde{e^{0,-}}\right)_{l} e^{i(n+1) \tau \beta_{l}^{-}}-\sum_{k=0}^{n}\left(\widetilde{\left(\xi^{k,+}\right.}\right)_{l} e^{i(n+1-k) \tau \beta_{l}^{+}}-\left(\widetilde{\xi^{k,-}}\right)_{l} e^{i(n+1-k) \tau \beta_{l}^{-}}\right) \\
& +\sum_{k=1}^{n}\left(\widetilde{W}_{l}^{k,+} e^{i(n+1-k) \tau \beta_{l}^{+}}-\widetilde{W}_{l}^{k,-} e^{i(n+1-k) \tau \beta_{l}^{-}}\right), \quad l \in \mathcal{T}_{M} .
\end{aligned}
$$

Using Cauchy inequality, we have

$$
\begin{aligned}
\left.\mid \widetilde{e^{n+1}}\right)\left._{l}\right|^{2} \leq & \left.\left.6\left(\left|\widetilde{\left(e^{0,+}\right)_{l}}\right|^{2}+\mid \widetilde{\left(e^{0,-}\right.}\right)_{l}\right|^{2}+(n+1) \sum_{k=0}^{n}\left|\widetilde{\left.\left(\widetilde{\xi^{k,+}}\right)_{l}\right|^{2}}+(n+1) \sum_{k=0}^{n}\right| \widetilde{\xi^{k,-}}\right)\left._{l}\right|^{2} \\
& \left.+n \sum_{k=1}^{n}\left|\widetilde{W}_{l}^{k,+}\right|^{2}+n \sum_{k=1}^{n}\left|\widetilde{W}_{l}^{k,-}\right|^{2}\right), \quad l \in \mathcal{T}_{M} .
\end{aligned}
$$

Then summing above inequalities together for $l \in \mathcal{T}_{M}$, making use of Lemma 3.4 and Parseval identity, 
noticing claim (3.31), for all $1 \leq n \leq \frac{T}{\tau}-1$, we have $\left.\left\|e^{n+1}(\cdot)\right\|_{L^{2}}=\frac{b-a}{2} \sum_{l=1}^{M-1} \mid \widetilde{\left(e^{n+1}\right.}\right)\left._{l}\right|^{2}$ and

$$
\begin{aligned}
\left\|e^{n+1}(\cdot)\right\|_{L^{2}}^{2} \leq & 6\left(\left\|e^{0,+}(\cdot)\right\|_{L^{2}}^{2}+\left\|e^{0,-}(\cdot)\right\|_{L^{2}}^{2}+(n+1) \sum_{k=0}^{n}\left(\left\|\xi^{k,+}(\cdot)\right\|_{L^{2}}^{2}+\left\|\xi^{k,-}(\cdot)\right\|_{L^{2}}^{2}\right)\right. \\
& \left.+n C_{0} \tau^{2} \sum_{k=0}^{n}\left(\left\|e^{k}(\cdot)\right\|_{L^{2}}^{2}+h^{2 m_{0}}\right)\right) \\
\leq & C_{1} h^{2 m_{0}}+(n+1)^{2} \tau^{2} C_{2}\left(\tau^{4}+h^{2 m}\right)+C_{0} T \tau \sum_{k=0}^{n}\left\|e^{k}(\cdot)\right\|_{L^{2}}^{2} \\
\leq & C_{3}\left(\tau^{4}+h^{2 m}\right)+C_{0} T \tau \sum_{k=0}^{n}\left\|e^{k}(\cdot)\right\|_{L^{2}}^{2}, \quad 1 \leq n \leq \frac{T}{\tau}-1,
\end{aligned}
$$

where $C_{0}, C_{1}, C_{2}$ and $C_{3}$ are constants depending on $T, f(\cdot), M_{1}$ and $\|\psi\|_{L^{\infty}\left([0, T] ; H_{s}^{m_{0}}\right)}$. Thus, the error bounds for $e^{0}(x)$ and $e^{1}(x)$ combined with discrete Gronwall inequality [4, 15, 16] would imply that there exists a $\tau_{2}>0$ independent of $\varepsilon$ such that when $0<\tau \leq \tau_{2}$, we can get

$$
\left\|e^{n+1}(\cdot)\right\|_{L^{2}}^{2} \lesssim \tau^{4}+h^{2 m}, \quad \text { and } \quad\left\|e^{n+1}(\cdot)\right\|_{L^{2}} \lesssim \tau^{2}+h^{m}, \quad 1 \leq n \leq \frac{T}{\tau}-1 .
$$

Then we estimate $\left\|\nabla e^{n+1}(\cdot)\right\|_{L^{2}}$. Similar as the above $L^{2}$ case, multiplying both sides of (3.33) by $\left|\mu_{l}\right|^{2}$ and summing up for all $l \in \mathcal{T}_{M}$, noticing claim (3.31), for all $1 \leq n \leq \frac{T}{\tau}-1$, using the derived $L^{2}$ estimates, we know

$$
\begin{aligned}
\left\|\nabla e^{n+1}(\cdot)\right\|_{L^{2}}^{2} \lesssim & \left(\left\|\nabla e^{0,+}(\cdot)\right\|_{L^{2}}^{2}+\left\|\nabla e^{0,-}(\cdot)\right\|_{L^{2}}^{2}+(n+1) \sum_{k=0}^{n}\left(\left\|\nabla \xi^{k,+}(\cdot)\right\|_{L^{2}}^{2}+\left\|\nabla \xi^{k,-}(\cdot)\right\|_{L^{2}}^{2}\right)\right. \\
& \left.+n \tau^{2} \sum_{k=0}^{n}\left(\left\|e^{k}(\cdot)\right\|_{L^{2}}^{2}+\left\|\nabla e^{k}(\cdot)\right\|_{L^{2}}^{2}+h^{2 m_{0}-2}\right)\right) \\
\lesssim & \left(\tau^{4}+h^{2 m-2}\right)+\tau \sum_{k=0}^{n}\left\|\nabla e^{k}(\cdot)\right\|_{L^{2}}^{2}, \quad 1 \leq n \leq \frac{T}{\tau}-1 .
\end{aligned}
$$

Again, the error bounds for $e^{0}(x)$ and $e^{1}(x)$ combined with the discrete Gronwall inequality [4, 15, 16, would imply that there exists a $\tau_{3}>0$ independent of $\varepsilon$ such that when $0<\tau \leq \tau_{3}$, we have

$$
\left\|\nabla e^{n+1}(\cdot)\right\|_{L^{2}}^{2} \lesssim \tau^{4}+h^{2 m-2}, \quad \text { and } \quad\left\|\nabla e^{n+1}(\cdot)\right\|_{L^{2}} \lesssim \tau^{2}+h^{m-1}, \quad 0 \leq n \leq \frac{T}{\tau}-1 .
$$

It remains to show the $l^{\infty}$ bound of $\psi^{n+1}$. The error bounds $\left\|e^{n+1}(\cdot)\right\|_{L^{2}}$ and $\left\|\nabla e^{n+1}(\cdot)\right\|_{L^{2}}$ and the discrete Sobolev inequality would imply that for some $h_{3}, \tau_{4}$ independent of $\varepsilon$, if $0<h \leq h_{3}, 0<\tau \leq \tau_{4}$

$$
\left\|e^{n+1}\right\|_{l^{\infty}} \lesssim \sqrt{\left\|e^{n+1}(\cdot)\right\|_{L^{2}}\left\|\nabla e^{n+1}(\cdot)\right\|_{L^{2}}} \lesssim h^{m-1}+\tau^{2}, \quad \text { and } \quad\left\|\psi^{n+1}\right\|_{l^{\infty}} \leq M_{1}+1,
$$

where $0 \leq n \leq \frac{T}{\tau}-1$. Now, in view of (3.21) and (3.36), we see

$$
\left\|\psi\left(\cdot, t_{n}\right)-\psi_{I}^{n}(\cdot)\right\|_{L^{2}} \lesssim h^{m}+\tau^{2}, \quad\left\|\nabla\left[\psi\left(\cdot, t_{n}\right)-\psi_{I}^{n}(\cdot)\right]\right\|_{L^{2}} \lesssim h^{m-1}+\tau^{2}, \quad 0 \leq n \leq \frac{T}{\tau},
$$

and this completes the proof, if we choose $0<\tau \leq \tau_{0}=\min \left\{\tau_{1}, \tau_{2}, \tau_{3}, \tau_{4}\right\}$ and $0<h \leq h_{0}=$ $\min \left\{h_{1}, h_{2}, h_{3}\right\}$. 
4. Convergence in the ill-prepared initial data case. Next, we start to prove Theorem 2.2 . Again we will treat $\mathcal{F}(z)$ as $\mathcal{F}_{B}(z)$ while not changing the notation. The idea of the proof is shown in the diagram (4.1), where $\psi_{h, \tau}$ denotes the EWI-SP numerical solution, $\psi$ is the exaction solution of the NLSW and $\psi^{s}$ is the exact solution of the corresponding NLS. In fact, the similar idea has been employed in the study of asymptotic preserving (AP) schemes [13, 19].

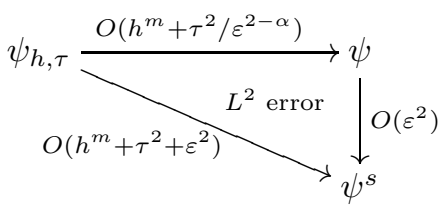

Proof of (2.37) in Theorem 2.2. First, let us consider (2.37), for which the proof is identical to the above proof for Theorem 2.2. The only thing needed to be modified is the local error bound, where $\left\|\xi^{n, \pm}(\cdot)\right\|_{L^{2}} \lesssim \tau\left(h^{m}+\varepsilon^{\alpha-2} \tau^{2}\right)$ and $\left\|\nabla \xi^{n, \pm}(\cdot)\right\|_{L^{2}} \lesssim \tau\left(h^{m-1}+\varepsilon^{\alpha-2} \tau^{2}\right)$ (Lemma 3.4) in this case. Following the analogous proof of Theorem 2.2, one can easily prove assertion (2.37) and we omit the details here for brevity.

Now, we come to prove (2.38), using a similar idea in 4. The proof is also similar to that of Theorem 2.2, and we just outline the main steps. Let $\psi^{s}:=\psi^{s}(x, t)$ be the solution of NLS (2.2), and we write $\psi^{s}\left(t_{n}\right)$ for $\psi^{s}\left(x, t_{n}\right)$ in short. Denote the 'error' $\mathcal{E}^{n} \in Y_{M}$ and $\mathcal{E}^{n}(x) \in X_{M}(n \geq 0)$ as

$$
\begin{aligned}
& \mathcal{E}_{j}^{n}=\left(P_{M} \psi^{s}\right)\left(x_{j}, t_{n}\right)-\psi_{j}^{n}, \quad j \in \mathcal{T}_{M}^{0}, \quad n \geq 0, \\
& \mathcal{E}^{n}(x)=I_{M}\left(\mathcal{E}^{n}\right)(x)=\sum_{l=1}^{M-1}\left(\widetilde{\mathcal{E}^{n}}\right)_{l} \Phi_{l}(x), \quad n \geq 0, \quad x \in \Omega .
\end{aligned}
$$

Using triangle inequality, we have for $n \geq 0$,

$$
\begin{aligned}
\left\|\psi\left(\cdot, t_{n}\right)-\psi_{I}^{n}(\cdot)\right\|_{L^{2}} & \leq\left\|\psi\left(\cdot, t_{n}\right)-\psi^{s}\left(\cdot, t_{n}\right)\right\|_{L^{2}}+\left\|\psi^{s}\left(\cdot, t_{n}\right)-P_{M}\left(\psi^{s}\left(t_{n}\right)\right)(\cdot)\right\|_{L^{2}}+\left\|\mathcal{E}^{n}(\cdot)\right\|_{L^{2}} \\
& \lesssim \varepsilon^{2}+h^{m_{0}}+\left\|\mathcal{E}^{n}(\cdot)\right\|_{L^{2}}, \\
\|(4.3) & \leq\left\|\nabla\left[\psi\left(\cdot, t_{n}\right)-\psi^{s}\left(\cdot, t_{n}\right)\right]\right\|_{L^{2}}+\left\|\nabla\left[\psi^{s}\left(\cdot, t_{n}\right)-P_{M}\left(\psi^{s}\left(t_{n}\right)\right)(\cdot)\right]\right\|_{L^{2}}+\left\|\nabla \mathcal{E}^{n}(\cdot)\right\|_{L^{2}} \\
\left\|\nabla\left[\psi\left(\cdot, t_{n}\right)-\psi_{I}^{n}(\cdot)\right]\right\|_{L^{2}} & \leq\left\|\varepsilon^{2}+h^{m_{0}}+\right\| \nabla \mathcal{E}^{n}(\cdot) \|_{L^{2}},
\end{aligned}
$$

which indicates that we only need to estimate $\left\|\mathcal{E}^{n}(\cdot)\right\|_{L^{2}}$ and $\left\|\nabla \mathcal{E}^{n}(\cdot)\right\|_{L^{2}}$.

In order to estimate the 'error' $\mathcal{E}^{n}$, we define the 'local truncation error' $\chi^{n}(x)=\sum_{l=1}^{M-1}\left(\widetilde{\chi^{n}}\right)_{l} \Phi_{l}(x) \in$ $X_{M}$ for $\psi^{s}(x, t)$ as

$$
\begin{aligned}
& \left.\widetilde{\left(\chi^{0}\right.}\right)_{l}=c_{l}^{0}\left(\widehat{\psi_{0}}\right)_{l}+d_{l}^{0}\left(\widehat{\psi_{1}}\right)_{l}+p_{l}^{0}\left(\widehat{\mathcal{F}\left(\psi_{0}\right)}\right)_{l}+q_{l}^{0}\left(\widehat{\left.\mathcal{D}\left(\widetilde{\psi^{s}(0)}\right)\right)}-\widehat{\left(\widehat{\psi^{s}(\tau)}\right)_{l}},\right. \\
& \left.\left.\left.\left(\widetilde{\chi^{n}}\right)_{l}=c_{l}\left(\psi^{s\left(t_{n-1}\right.}\right)\right)_{l}+d_{l}\left(\widehat{\psi^{s}\left(t_{n}\right)}\right)_{l}+p_{l}\left(\widetilde{\mathcal{F}\left(\psi^{s}\left(t_{n}\right)\right.}\right)\right)_{l}+q_{l}\left(\widehat{\mathcal{D}\left(\psi^{s}\left(t_{n}\right)\right.}\right)\right)_{l}-p_{l}^{*}\left(\mathcal { F } \left(\widetilde{\left.\left.\psi^{s}\left(t_{n-1}\right)\right)\right)_{l}}\right.\right. \\
& \left.-q_{l}^{*}\left(\mathcal{D}\left(\widehat{\psi^{s}\left(t_{n-1}\right.}\right)\right)\right)-\left(\widehat{\left.\psi^{s\left(t_{n+1}\right.}\right)}\right)_{l}, \quad n \geq 1 \text {. }
\end{aligned}
$$

Rewriting the NLS (2.2) as

$$
i \partial_{t} \psi^{s}-\varepsilon^{2} \partial_{t t} \psi^{s}+\partial_{x x} \psi^{s}+\left(f\left(\left|\psi^{s}\right|^{2}\right) \psi^{s}+\varepsilon^{2} \partial_{t t} \psi^{s}\right)=0,
$$


and recalling $\psi^{s}(x, 0)=\psi_{0}$ and $\partial_{t} \psi^{s}(x, 0)=\psi_{1}(x)$, we can prove analogous results in the same way as Lemma 3.4. Here we present the results and omit the details.

Lemma 4.1. Let $\chi^{n}(x)(n \geq 0)$ be defined as 4.5), $f(\cdot) \in C^{k}([0, \infty))(k \geq 3)$, under assumptions (A) and (B), we have the following decomposition of $\chi^{n}(x)(n \geq 0)$ (3.12),

$$
\begin{aligned}
& \chi^{0}(x)=e^{i \tau \beta_{l}^{+}} \chi^{0,+}(x)-e^{i \tau \beta_{l}^{-}} \chi^{0,-}(x), \\
& \chi^{n}(x)=e^{i \tau \beta_{l}^{+}} \chi^{n,+}(x)-e^{i \tau \beta_{l}^{-}} \chi^{n,-}(x)-e^{\frac{i \tau}{\varepsilon^{2}}}\left(\chi^{n-1,+}(x)-\chi^{n-1,-}(x)\right), \quad n \geq 1,
\end{aligned}
$$

where $\chi^{n, \pm}(x)=\sum_{l=1}^{M-1}\left(\widetilde{\chi^{n, \pm}}\right)_{l} \Phi_{l}(x) \in X_{M}$ and for $\alpha \in[0,2)$, i.e. the ill-prepared initial data case, we have

$$
\left\|\chi^{n, \pm}(\cdot)\right\|_{L^{2}} \lesssim \tau h^{m}+\tau^{3}+\tau \varepsilon^{2}, \quad\left\|\nabla \chi^{n, \pm}(\cdot)\right\|_{L^{2}} \lesssim \tau h^{m-1}+\tau^{3}+\tau \varepsilon^{2}, \quad m=\min \left\{m_{0}, k\right\}, \quad n \geq 0 .
$$

The decomposition in Lemma 4.1 is analogous to that in the proof of Lemma 3.4 and the lemma also holds true for $\alpha \geq 2$.

Proof of (2.38) and (2.39) in Theorem [2.2. First, for $\mathcal{E}^{0}(x)$ and $\mathcal{E}^{1}(x)$ we note that we can write

$$
\left(\widetilde{\mathcal{E}^{0}}\right)_{l}=\left(\widetilde{\mathcal{E}^{0,+}}\right)_{l}+\left(\widetilde{\mathcal{E}^{0,-}}\right)_{l}, \quad\left(\widetilde{\mathcal{E}^{1}}\right)_{l}=\left(\widetilde{\mathcal{E}^{0,+}}\right)_{l} e^{i \tau \beta_{l}^{+}}+\left(\widetilde{\mathcal{E}^{0,-}}\right)_{l} e^{i \beta_{l}^{-}}-\left(\widetilde{\chi^{0}}\right)_{l}, \quad l \in \mathcal{T}_{M},
$$

where $\mathcal{E}^{0, \pm}(x)=\sum_{l=1}^{M-1}\left(\widetilde{\mathcal{E}^{0, \pm}}\right)_{l} \Phi_{l}(x) \in X_{M}$ are given by

$$
\left(\widetilde{\mathcal{E}^{0,+}}\right)_{l}=-\frac{\beta_{l}^{-}}{\beta_{l}}\left[\left(\widehat{\psi_{0}}\right)_{l}-\left(\widetilde{\psi_{0}}\right)_{l}\right]-\frac{i}{\beta_{l}}\left[\left(\widehat{\psi_{1}}\right)_{l}-\left(\widetilde{\psi_{1}^{\varepsilon}}\right)_{l}\right], \quad\left(\widetilde{\mathcal{E}^{0,-}}\right)_{l}=\frac{\beta_{l}^{+}}{\beta_{l}}\left[\left(\widehat{\psi_{0}}\right)_{l}-\left(\widetilde{\psi_{0}}\right)_{l}\right]+\frac{i}{\beta_{l}}\left[\left(\widehat{\psi_{1}}\right)_{l}-\left(\widetilde{\psi_{1}^{\varepsilon}}\right)_{l}\right] .
$$

In view of the fact that $\frac{1}{\beta_{l}} \leq \varepsilon^{2}$ and $\psi_{1}^{\varepsilon}=\psi_{1}+\varepsilon^{\alpha} \omega^{\varepsilon}(x)$, it is easy to verify that

$$
\left\|\mathcal{E}^{0, \pm}(\cdot)\right\|_{L^{2}} \lesssim h^{m}+\varepsilon^{2+\alpha}, \quad\left\|\nabla \mathcal{E}^{0, \pm}(\cdot)\right\|_{L^{2}} \lesssim h^{m-1}+\varepsilon^{2+\alpha},
$$

which implies (2.38) for $n=0,1$ in view of Lemma 4.1. For time steps $n \geq 2$, following the same procedure of the proof for Theorem 2.1 we can get conclusion (2.38) for all $0 \leq n \leq \frac{T}{\tau}$ with the help of Lemma 4.1 .

Finally, it remains to prove (2.39) which enables us to simplify the nonlinearity $\mathcal{F}$ to $\mathcal{F}_{B}$. Since assertions (2.37) and (2.38) have been proven and the constants in the estimates are independent of $\varepsilon$, $\tau$ and $h$, we take the minimum of $\varepsilon^{2}$ and $\frac{\tau^{2}}{\varepsilon^{2-\alpha}}$ for $0<\varepsilon \leq 1$, and the following holds for $0 \leq n \leq \frac{T}{\tau}$,

$$
\left\|\psi\left(\cdot, t_{n}\right)-\psi_{I}^{n}(\cdot)\right\|_{L^{2}} \lesssim h^{m}+\tau^{4 /(2-\alpha)}, \quad\left\|\nabla\left[\psi\left(\cdot, t_{n}\right)-\psi_{I}^{n}(\cdot)\right]\right\|_{L^{2}} \lesssim h^{m-1}+\tau^{4 /(2-\alpha)} .
$$

Hence for $\alpha \in[0,2)$, Sobolev inequality implies that

$$
\left\|\psi\left(\cdot, t_{n}\right)-\psi_{I}^{n}(\cdot)\right\|_{L^{\infty}} \leq \sqrt{\left\|\psi\left(\cdot, t_{n}\right)-\psi_{I}^{n}(\cdot)\right\|_{L^{2}}\left\|\nabla\left[\psi\left(\cdot, t_{n}\right)-\psi_{I}^{n}(\cdot)\right]\right\|_{L^{2}}} \lesssim h^{m-1}+\tau,
$$

which justifies $\max _{j \in \mathcal{T}_{M}}\left|\psi\left(x_{j}, t_{n}\right)-\psi_{j}^{n}\right| \leq 1$ for sufficient small $\tau$ and $h$. Hence (2.39) is proven. The proof of Theorem 2.2 is complete.

REMARK 4.1. Let us make a final remark that the above proof and the main results (Theorem 2.1 and (2.2) are valid for $2 D$ and $3 D$ cases. The key point for extension to $2 D$ and $3 D$ is the discrete Sobolev inequality in higher dimensions as [28]

$$
\left\|\psi_{h}\right\|_{\infty} \leq C|\ln h|\left\|\psi_{h}\right\|_{H^{1}}, \quad\left\|\phi_{h}\right\|_{\infty} \leq C h^{-1 / 2}\left\|\phi_{h}\right\|_{H^{1}},
$$


where $\psi_{h}$ and $\phi_{h}$ are $2 D$ and $3 D$ mesh functions with zero at the boundary, respectively, and the discrete semi- $H^{1}$ norm $\|\cdot\|_{H^{1}}$ and $l^{\infty}$ norm $\|\cdot\|_{\infty}$ can be defined similarly as the $1 D$ version (2.34). Thus, by requiring the time step $\tau$ satisfies the additional condition $\tau \lesssim h$, with the discrete Sobolev inequality and the uniform bounds for the semi- $H^{1}$ norm at $h^{m-1}+\tau$, using $m \geq 2$, we can control the $l^{\infty}$ bound of the numerical solution, which guarantees the correctness of the cutoff (3.19). The readers can refer to [4] for more discussion.

5. Numerical results. In this section, we report the numerical results of our scheme EWI-SP (2.28)-(2.29) to confirm our theoretical analysis. The nonlinearity is taken as $f\left(|z|^{2}\right)=-|z|^{2}$.

\begin{tabular}{l|c|c|c|c|c|c|c|c}
\hline & \multicolumn{5}{|c|}{$\alpha=0$} & \multicolumn{4}{c}{$\alpha=2$} \\
\cline { 2 - 9 } & $h=2$ & $h=1$ & $h=1 / 2$ & $h=1 / 4$ & $h=2$ & $h=1$ & $h=1 / 2$ & $h=1 / 4$ \\
\hline$\varepsilon=1 / 2$ & $1.03 \mathrm{E} 00$ & $9.59 \mathrm{E}-2$ & $6.76 \mathrm{E}-4$ & $4.15 \mathrm{E}-8$ & $9.45 \mathrm{E}-1$ & $8.85 \mathrm{E}-2$ & $6.39 \mathrm{E}-4$ & $3.90 \mathrm{E}-8$ \\
$\varepsilon=1 / 2^{2}$ & $8.85 \mathrm{E}-1$ & $6.85 \mathrm{E}-2$ & $2.36 \mathrm{E}-4$ & $2.47 \mathrm{E}-9$ & $8.55 \mathrm{E}-1$ & $6.96 \mathrm{E}-2$ & $2.45 \mathrm{E}-4$ & $2.43 \mathrm{E}-9$ \\
$\varepsilon=1 / 2^{3}$ & $8.62 \mathrm{E}-1$ & $6.71 \mathrm{E}-2$ & $1.57 \mathrm{E}-4$ & $1.30 \mathrm{E}-10$ & $8.55 \mathrm{E}-1$ & $6.72 \mathrm{E}-2$ & $1.57 \mathrm{E}-4$ & $1.39 \mathrm{E}-10$ \\
$\varepsilon=1 / 2^{4}$ & $8.57 \mathrm{E}-1$ & $6.71 \mathrm{E}-2$ & $1.40 \mathrm{E}-4$ & $7.00 \mathrm{E}-11$ & $8.60 \mathrm{E}-1$ & $6.72 \mathrm{E}-2$ & $1.40 \mathrm{E}-4$ & $6.99 \mathrm{E}-11$ \\
$\varepsilon=1 / 2^{5}$ & $8.61 \mathrm{E}-1$ & $6.73 \mathrm{E}-2$ & $1.37 \mathrm{E}-4$ & $5.44 \mathrm{E}-11$ & $8.62 \mathrm{E}-1$ & $6.72 \mathrm{E}-2$ & $1.37 \mathrm{E}-4$ & $5.45 \mathrm{E}-11$ \\
$\varepsilon=1 / 2^{6}$ & $8.62 \mathrm{E}-1$ & $6.73 \mathrm{E}-2$ & $1.36 \mathrm{E}-4$ & $5.14 \mathrm{E}-11$ & $8.62 \mathrm{E}-1$ & $6.73 \mathrm{E}-2$ & $1.36 \mathrm{E}-4$ & $5.15 \mathrm{E}-11$ \\
$\varepsilon=1 / 2^{10}$ & $8.62 \mathrm{E}-1$ & $6.73 \mathrm{E}-2$ & $1.36 \mathrm{E}-4$ & $5.06 \mathrm{E}-11$ & $8.62 \mathrm{E}-1$ & $6.73 \mathrm{E}-2$ & $1.36 \mathrm{E}-4$ & $5.06 \mathrm{E}-11$ \\
$\varepsilon=1 / 2^{20}$ & $8.62 \mathrm{E}-1$ & $6.73 \mathrm{E}-2$ & $1.36 \mathrm{E}-4$ & $5.06 \mathrm{E}-11$ & $8.62 \mathrm{E}-1$ & $6.73 \mathrm{E}-2$ & $1.36 \mathrm{E}-4$ & $5.06 \mathrm{E}-11$ \\
\hline
\end{tabular}

Spatial error analysis for EWI-SP [2.28)- 2.29), with different $\varepsilon$ for Case $I(\alpha=2)$ and Case II $(\alpha=0)$, for $\left\|e^{n}(x)\right\|_{H^{1}}$.

\begin{tabular}{ccccccccc}
\hline$\alpha=2$ & $\tau=0.2$ & $\tau=\frac{0.2}{4}$ & $\tau=\frac{0.2}{4^{2}}$ & $\tau=\frac{0.2}{4^{3}}$ & $\tau=\frac{0.2}{4^{4}}$ & $\tau=\frac{0.2}{4^{5}}$ & $\tau=\frac{0.2}{4^{6}}$ & $\tau=\frac{0.2}{4^{7}}$ \\
\hline$\varepsilon=1 / 2$ & $4.63 \mathrm{E}-2$ & $2.97 \mathrm{E}-3$ & $1.87 \mathrm{E}-4$ & $1.17 \mathrm{E}-5$ & $7.34 \mathrm{E}-7$ & $4.59 \mathrm{E}-8$ & $2.87 \mathrm{E}-9$ & $1.87 \mathrm{E}-10$ \\
rate & - & 1.98 & 1.99 & 2.00 & 2.00 & 2.00 & 2.00 & 1.97 \\
\hline$\varepsilon=1 / 2^{2}$ & $4.22 \mathrm{E}-2$ & $4.52 \mathrm{E}-3$ & $2.83 \mathrm{E}-4$ & $1.77 \mathrm{E}-5$ & $1.11 \mathrm{E}-6$ & $6.91 \mathrm{E}-8$ & $4.32 \mathrm{E}-9$ & $2.77 \mathrm{E}-10$ \\
rate & - & 1.61 & 2.00 & 2.00 & 2.00 & 2.00 & 2.00 & 1.98 \\
\hline$\varepsilon=1 / 2^{3}$ & $5.01 \mathrm{E}-2$ & $3.99 \mathrm{E}-3$ & $3.76 \mathrm{E}-4$ & $2.37 \mathrm{E}-5$ & $1.48 \mathrm{E}-6$ & $9.27 \mathrm{E}-8$ & $5.78 \mathrm{E}-9$ & $3.53 \mathrm{E}-10$ \\
rate & - & 1.83 & 1.70 & 2.00 & 2.00 & 2.00 & 2.00 & 2.02 \\
\hline$\varepsilon=1 / 2^{4}$ & $5.50 \mathrm{E}-2$ & $3.73 \mathrm{E}-3$ & $3.09 \mathrm{E}-4$ & $2.45 \mathrm{E}-5$ & $1.53 \mathrm{E}-6$ & $9.61 \mathrm{E}-8$ & $6.01 \mathrm{E}-9$ & $3.74 \mathrm{E}-10$ \\
rate & - & 1.94 & 1.80 & 1.83 & 2.00 & 2.00 & 2.00 & 2.00 \\
\hline$\varepsilon=1 / 2^{5}$ & $5.65 \mathrm{E}-2$ & $3.85 \mathrm{E}-3$ & $2.43 \mathrm{E}-4$ & $1.95 \mathrm{E}-5$ & $1.61 \mathrm{E}-6$ & $1.02 \mathrm{E}-7$ & $6.38 \mathrm{E}-9$ & $4.04 \mathrm{E}-10$ \\
rate & - & 1.94 & 1.99 & 1.82 & 1.80 & 1.99 & 2.00 & 1.99 \\
\hline$\varepsilon=1 / 2^{6}$ & $5.69 \mathrm{E}-2$ & $3.88 \mathrm{E}-3$ & $2.45 \mathrm{E}-4$ & $1.54 \mathrm{E}-5$ & $1.25 \mathrm{E}-6$ & $1.00 \mathrm{E}-7$ & $6.30 \mathrm{E}-9$ & $3.82 \mathrm{E}-10$ \\
rate & - & 1.94 & 1.99 & 2.00 & 1.81 & 1.82 & 1.99 & 2.02 \\
\hline$\varepsilon=1 / 2^{10}$ & $5.70 \mathrm{E}-2$ & $3.89 \mathrm{E}-3$ & $2.46 \mathrm{E}-4$ & $1.54 \mathrm{E}-5$ & $9.62 \mathrm{E}-7$ & $6.01 \mathrm{E}-8$ & $3.76 \mathrm{E}-9$ & $2.48 \mathrm{E}-10$ \\
rate & - & 1.94 & 1.99 & 2.00 & 2.00 & 2.00 & 2.00 & 1.96 \\
\hline$\varepsilon=1 / 2^{20}$ & $5.70 \mathrm{E}-2$ & $3.89 \mathrm{E}-3$ & $2.46 \mathrm{E}-4$ & $1.54 \mathrm{E}-5$ & $9.62 \mathrm{E}-7$ & $6.01 \mathrm{E}-8$ & $3.76 \mathrm{E}-9$ & $2.46 \mathrm{E}-10$ \\
rate & - & 1.94 & 1.99 & 2.00 & 2.00 & 2.00 & 2.00 & 1.97 \\
\hline
\end{tabular}

Temporal error analysis for $E W I-S P$, with different $\varepsilon$ for Case $I(\alpha=2)$, with $\left\|e^{n}(\cdot)\right\|_{H^{1}}$. The convergence rate is calculated as $\log _{2}\left(\left\|e^{n}(\cdot, 4 \tau)\right\|_{H^{1}} /\left\|e^{n}(\cdot, \tau)\right\|_{H^{1}}\right) / 2$. 
Optimal uniform error estimates of EWI-SP for NLSW

\begin{tabular}{ccccccccc}
\hline$\alpha=0$ & $\tau=0.2$ & $\tau=\frac{0.2}{4}$ & $\tau=\frac{0.2}{4^{2}}$ & $\tau=\frac{0.2}{4}$ & $\tau=\frac{0.2}{4}$ & $\tau=\frac{0.2}{4^{5}}$ & $\tau=\frac{0.2}{46}$ & $\tau=\frac{0.2}{47}$ \\
\hline$\varepsilon=1 / 2$ & $1.22 \mathrm{E}-1$ & $7.58 \mathrm{E}-3$ & $4.75 \mathrm{E}-4$ & $2.97 \mathrm{E}-5$ & $1.86 \mathrm{E}-6$ & $1.16 \mathrm{E}-7$ & $7.24 \mathrm{E}-9$ & $4.42 \mathrm{E}-10$ \\
rate & - & 2.00 & 2.00 & 2.00 & 2.00 & 2.00 & 2.00 & 2.02 \\
\hline$\varepsilon=1 / 2^{2}$ & $2.16 \mathrm{E}-1$ & $2.61 \mathrm{E}-2$ & $1.63 \mathrm{E}-3$ & $1.02 \mathrm{E}-4$ & $6.36 \mathrm{E}-6$ & $3.98 \mathrm{E}-7$ & $2.48 \mathrm{E}-8$ & $1.55 \mathrm{E}-9$ \\
rate & - & 1.52 & 2.00 & 2.00 & 2.00 & 2.00 & 2.00 & 2.00 \\
\hline$\varepsilon=1 / 2^{3}$ & $1.34 \mathrm{E}-1$ & $6.07 \mathrm{E}-2$ & $6.17 \mathrm{E}-3$ & $3.89 \mathrm{E}-4$ & $2.44 \mathrm{E}-5$ & $1.52 \mathrm{E}-6$ & $9.52 \mathrm{E}-8$ & $5.91 \mathrm{E}-9$ \\
rate & - & 0.57 & 1.65 & 1.99 & 2.00 & 2.00 & 2.00 & 2.00 \\
\hline$\varepsilon=1 / 2^{4}$ & $1.31 \mathrm{E}-1$ & $1.12 \mathrm{E}-2$ & $1.57 \mathrm{E}-2$ & $1.54 \mathrm{E}-3$ & $9.64 \mathrm{E}-5$ & $6.04 \mathrm{E}-6$ & $3.77 \mathrm{E}-7$ & $2.34 \mathrm{E}-8$ \\
rate & - & 1.73 & -0.20 & 1.67 & 2.00 & 2.00 & 2.00 & 2.00 \\
\hline$\varepsilon=1 / 2^{5}$ & $1.32 \mathrm{E}-1$ & $8.47 \mathrm{E}-3$ & $1.66 \mathrm{E}-3$ & $3.91 \mathrm{E}-3$ & $3.86 \mathrm{E}-4$ & $2.41 \mathrm{E}-5$ & $1.51 \mathrm{E}-6$ & $9.37 \mathrm{E}-8$ \\
rate & - & 1.98 & 1.18 & -0.62 & 1.67 & 2.00 & 2.00 & 2.01 \\
\hline$\varepsilon=1 / 2^{6}$ & $1.33 \mathrm{E}-1$ & $8.09 \mathrm{E}-3$ & $6.63 \mathrm{E}-4$ & $3.61 \mathrm{E}-4$ & $9.87 \mathrm{E}-4$ & $9.66 \mathrm{E}-5$ & $6.02 \mathrm{E}-6$ & $3.75 \mathrm{E}-7$ \\
rate & - & 2.02 & 1.80 & 0.44 & -0.73 & 1.68 & 2.00 & 2.00 \\
\hline$\varepsilon=1 / 2^{7}$ & $1.34 \mathrm{E}-1$ & $8.05 \mathrm{E}-3$ & $5.01 \mathrm{E}-4$ & $9.12 \mathrm{E}-5$ & $8.78 \mathrm{E}-5$ & $2.47 \mathrm{E}-4$ & $2.42 \mathrm{E}-5$ & $1.50 \mathrm{E}-6$ \\
rate & - & 2.03 & 2.00 & 1.23 & 0.03 & -0.75 & 1.68 & 2.01 \\
\hline$\varepsilon=1 / 2^{8}$ & $1.34 \mathrm{E}-1$ & $8.05 \mathrm{E}-3$ & $5.00 \mathrm{E}-4$ & $3.64 \mathrm{E}-5$ & $2.16 \mathrm{E}-5$ & $2.20 \mathrm{E}-5$ & $6.19 \mathrm{E}-5$ & $6.01 \mathrm{E}-6$ \\
rate & - & 2.03 & 2.00 & 1.89 & 0.38 & -0.01 & -0.75 & 1.68 \\
\hline$\varepsilon=1 / 2^{10}$ & $1.34 \mathrm{E}-1$ & $8.06 \mathrm{E}-3$ & $5.03 \mathrm{E}-4$ & $3.17 \mathrm{E}-5$ & $2.97 \mathrm{E}-6$ & $2.02 \mathrm{E}-6$ & $1.97 \mathrm{E}-6$ & $1.99 \mathrm{E}-6$ \\
rate & - & 2.03 & 2.00 & 1.99 & 1.71 & 0.28 & 0.02 & -0.01 \\
\hline$\varepsilon=1 / 2^{20}$ & $1.34 \mathrm{E}-1$ & $8.06 \mathrm{E}-3$ & $5.02 \mathrm{E}-4$ & $3.14 \mathrm{E}-5$ & $1.96 \mathrm{E}-6$ & $1.23 \mathrm{E}-7$ & $7.66 \mathrm{E}-9$ & $4.80 \mathrm{E}-10$ \\
rate & - & 2.03 & 2.00 & 2.00 & 2.00 & 2.00 & 2.00 & 2.00 \\
\hline & & & & TABLE 5.3 & & & \\
\end{tabular}

Temporal error analysis for EWI-SP, with different $\varepsilon$ for Case $I I(\alpha=0)$, with $\left\|e^{n}(\cdot)\right\|_{H^{1}}$. The convergence rate is calculated as $\log _{2}\left(\left\|e^{n}(\cdot, 4 \tau)\right\|_{H^{1}} /\left\|e^{n}(\cdot, \tau)\right\|_{H^{1}}\right) / 2$.

\begin{tabular}{ccccccc}
\hline \multirow{2}{*}{$\alpha=0$} & $\varepsilon=0.5$ & $\varepsilon=0.5 / 2$ & $\varepsilon=0.5 / 2^{2}$ & $\varepsilon=0.5 / 2^{3}$ & $\varepsilon=0.5 / 2^{4}$ & $\varepsilon=0.5 / 2^{5}$ \\
& $\tau=0.2$ & $\tau=0.2 / 4$ & $\tau=0.2 / 4^{2}$ & $\tau=0.2 / 4^{3}$ & $\tau=0.2 / 4^{4}$ & $\tau=0.2 / 4^{5}$ \\
\hline$\left\|e^{n}\right\|_{H^{1}}$ & $1.22 \mathrm{E}-1$ & $2.61 \mathrm{E}-2$ & $6.17 \mathrm{E}-3$ & $1.54 \mathrm{E}-3$ & $3.86 \mathrm{E}-4$ & $9.66 \mathrm{E}-5$ \\
rate & - & 1.11 & 1.04 & 1.00 & 1.00 & 1.00 \\
\hline \multicolumn{7}{c}{$\begin{array}{c}\text { Degeneracy of convergence rate for } \\
\log _{2}\left(\left\|e^{n}\left(2^{2} \tau, 2 \varepsilon\right)\right\|_{H^{1}} /\left\|e^{n}(\tau, \varepsilon)\right\|_{H^{1}}\right) / 2 .\end{array}$}
\end{tabular}

For the numerical experiments, the initial value is chosen as $\psi_{0}(x)=\pi^{-1 / 4} e^{-x^{2} / 2}$ and $\omega^{\varepsilon}(x)=$ $e^{-x^{2} / 2}$ in (2.1). The computational domain is chosen as $[a, b]=[-16,16]$. The 'exact' solution is computed using the proposed scheme with very fine mesh $h=1 / 128$ and time step $\tau=10^{-6}$. We study the following two cases of initial data:

Case I. $\alpha=2$, i.e., the well-prepared initial data case.

Case II. $\alpha=0$, i.e., the ill-prepared initial data case.

The errors are defined as $e^{n} \in Y_{M}$ and $e^{n}(x) \in X_{M}$ with $e_{j}^{n}=\psi\left(x_{j}, t_{n}\right)-\psi_{j}^{n}$ and $e^{n}(x)=$ $\psi\left(t_{n}\right)-I_{M}\left(\psi^{n}\right)(x)$. We measure the $H^{1}$ norm of $e^{n}(x)$, i.e. $\left\|e^{n}(\cdot)\right\|_{H^{1}}=\left\|e^{n}(\cdot)\right\|_{L^{2}}+\left\|\nabla e^{n}(\cdot)\right\|_{L^{2}}$.

The errors are displayed at $t=1$. For spatial error analysis, we choose time step $\tau=10^{-6}$, such that the error in time discretization can be neglected (cf. Tab. 5.1). For temporal error analysis, we choose $h=1 / 32$ such that the spatial error can be ignored. 
Tab. 5.1 depicts the spatial errors for both Case I and Case II, which clearly demonstrates that EWI-SP is uniformly spectral accurate in $h$ for all $\varepsilon \in(0,1]$. Tabs. 5.2 and 5.3 present the temporal errors for Case $I$ and $I I$, respectively. From Tab. [5.2, we can conclude that the temporal error of EWI-SP is of second order uniformly in $\varepsilon \in(0,1]$, for $\alpha=2$. From Tab. 5.3 with $\alpha=0$, when time step $\tau$ is small (upper triangle part of the table), second order convergence of the temporal error is clear; when $\varepsilon$ is small (lower triangle part of the table), second order convergence of the temporal error is also clear; near the diagonal part where $\tau \sim \varepsilon^{2}$, degeneracy of the convergence rate for the temporal error is observed. Tab 5.4 lists the degenerate convergence rate for the temporal error with $\alpha=0$ at the parameter regime $\tau \sim \varepsilon^{2}$, predicted by our error estimates in Theorem 2.2. Numerical results clearly confirms that the temporal error of EWI-SP is of $O\left(\tau^{2}\right)$ and $O(\tau)$ uniformly in $\varepsilon \in(0,1]$ for $\alpha=2$ and $\alpha=0$, respectively, while EWI-SP is uniformly spectral accurate in mesh size $h$ for $\varepsilon \in(0,1]$, for both well-prepared case $\alpha=2$ and ill-prepared case $\alpha=0$.

6. Conclusion. We have proposed and analyzed an exponential wave integrator sine pseudospectral (EWI-SP) method for the nonlinear Schrödinger equation perturbed by the wave operator (NLSW) in one, two and three dimensions, where a small dimensionless parameter $\varepsilon \in(0,1]$ is used to describe the perturbation strength. The difficulty of the problem is that the solution of NLSW oscillates in time at $O\left(\varepsilon^{2}\right)$ wavelength with $O\left(\varepsilon^{4}\right)$ and $O\left(\varepsilon^{2}\right)$ amplitude for well-prepared and ill-prepared initial data, respectively, especially for $0<\varepsilon \ll 1$. We have proved the uniform spectral accuracy of EWI-SP in mesh size $h$, and uniform convergence rates of EWI-SP in time step $\tau$ at the order $O\left(\tau^{2}\right)$ and $O(\tau)$ for well-prepared and ill-prepared initial data, respectively, in $L^{2}$ norm and semi- $H^{1}$ norm. This improves the convergence results of finite difference methods for NLSW in [4. Numerical results suggeste the error estimates are optimal.

Acknowledgments. Part of the work was done while the authors were visiting the Institute for Mathematical Sciences, National University of Singapore, in 2011.

\section{Appendix: proof of the claim (3.31)}

We notice that $\mathcal{F}$ is assumed to enjoy the properties of $\mathcal{F}_{B}(3.19)$. Employing Cauchy inequality and the fact about the coefficients $\left|p_{l}^{ \pm}\right| \lesssim \tau,\left|q_{l}^{ \pm}\right| \lesssim \tau^{2}$ (Lemma 3.4), we can find

$$
\left.\left.\sum_{l=1}^{M-1}\left|\widetilde{W}_{l}^{n, \pm}\right|^{2} \lesssim \tau^{2}\left[\sum_{l=1}^{M-1} \mid\left(\widetilde{\mathcal{F}\left(\psi\left(t_{n}\right)\right.}\right)\right)_{l}-\left.\left(\widetilde{\mathcal{F}\left(\psi^{n}\right)}\right)_{l}\right|^{2}+\tau^{2} \sum_{l=1}^{M-1} \mid \delta_{t}^{-}\left(\widetilde{\mathcal{F}\left(\psi\left(t_{n}\right)\right.}\right)\right)_{l}-\left.\delta_{t}^{-}\left(\widetilde{\mathcal{F}\left(\psi^{n}\right)}\right)_{l}\right|^{2}\right]
$$

Estimating each term on the RHS above, noticing the assumption which implies $\mathcal{F}(\cdot)$ is global Lipschitz, we get

$$
\begin{aligned}
& \left.\frac{b-a}{2} \sum_{l=1}^{M-1} \mid\left(\widetilde{\mathcal{F}\left(\psi\left(t_{n}\right)\right.}\right)\right)_{l}-\left.\left(\widetilde{\mathcal{F}\left(\psi^{n}\right)}\right)_{l}\right|^{2}=\| I_{M}\left(\mathcal{F}\left(\psi\left(t_{n}\right)\right)(\cdot)-I_{M}\left(\mathcal{F}\left(\psi^{n}\right)\right)(\cdot) \|_{L^{2}}^{2}\right. \\
& =h \sum_{j=1}^{M-1}\left|\mathcal{F}\left(\psi\left(x_{j}, t_{n}\right)\right)-\mathcal{F}\left(\psi_{j}^{n}\right)\right|^{2} \leq C_{\mathcal{F}} h \sum_{j=1}^{M-1}\left|\psi\left(x_{j}, t_{n}\right)-\psi_{j}^{n}\right|^{2}=C_{\mathcal{F}}\left\|I_{M}\left(\psi\left(t_{n}\right)\right)(\cdot)-I_{M}\left(\psi^{n}\right)(\cdot)\right\|_{L^{2}}^{2} \\
& \leq 2 C_{\mathcal{F}}\left(\left\|I_{M}\left(\psi\left(t_{n}\right)\right)(\cdot)-P_{M}\left(\psi\left(t_{n}\right)\right)(\cdot)\right\|_{L^{2}}^{2}+\left\|P_{M}\left(\psi\left(t_{n}\right)\right)(\cdot)-I_{M}\left(\psi^{n}\right)(\cdot)\right\|_{L^{2}}^{2}\right) \lesssim\left\|e^{n}(\cdot)\right\|_{L^{2}}^{2}+h^{2 m_{0}},
\end{aligned}
$$


where $C_{\mathcal{F}}$ only depends on $f(\cdot)$ and $M_{1}$. Similarly, making use of the properties of $\mathcal{F}$, we have

$$
\begin{aligned}
& \frac{b-a}{2} \sum_{l=1}^{M-1} \tau^{2} \mid \delta_{t}^{-}\left(\widetilde{\left.\mathcal{F}\left(\psi\left(t_{n}\right)\right)\right)_{l}}-\left.\delta_{t}^{-}\left(\widetilde{\mathcal{F}\left(\psi^{n}\right)}\right)_{l}\right|^{2}=h \tau^{2} \sum_{j=1}^{M-1}\left|\delta_{t}^{-} \mathcal{F}\left(\psi\left(x_{j}, t_{n}\right)\right)-\delta_{t}^{-} \mathcal{F}\left(\psi_{j}^{n}\right)\right|^{2}\right. \\
& \leq \widetilde{C}_{\mathcal{F}} h\left(\sum_{j=1}^{M-1}\left|\psi\left(x_{j}, t_{n}\right)-\psi_{j}^{n}\right|^{2}+\sum_{j=1}^{M-1}\left|\psi\left(x_{j}, t_{n-1}\right)-\psi_{j}^{n-1}\right|^{2}\right) \leq \widetilde{C}_{\mathcal{F}} h \sum_{k=n-1}^{n} \sum_{j=1}^{M-1}\left|\psi\left(x_{j}, t_{k}\right)-\psi_{j}^{k}\right|^{2} \\
& =\widetilde{C}_{\mathcal{F}} \sum_{k=n-1}^{n}\left\|I_{M}\left(\psi\left(t_{k}\right)\right)(\cdot)-I_{M}\left(\psi^{k}\right)(\cdot)\right\|_{L^{2}}^{2} \lesssim\left\|e^{n}(\cdot)\right\|_{L^{2}}^{2}+\left\|e^{n-1}(\cdot)\right\|_{L^{2}}^{2}+h^{2 m_{0}},
\end{aligned}
$$

where $\widetilde{C}_{\mathcal{F}}$ only depends on $f(\cdot)$ and $M_{1}$. Combining all the above results together, we get the first conclusion in the claim (3.31). Similarly, using Lemma 3.2, we can get

$$
\sum_{l=1}^{M-1}\left|\mu_{l}\right|^{2}\left|\widetilde{W}_{l}^{n, \pm}\right|^{2} \lesssim \tau^{2}\left[\left\|\nabla\left[I_{M}\left(\mathcal{F}\left(\psi\left(t_{n}\right)\right)-\mathcal{F}\left(\psi^{n}\right)\right)\right]\right\|_{L^{2}}^{2}+\tau^{2}\left\|\nabla\left[I_{M}\left(\delta_{t}^{-} \mathcal{F}\left(\psi\left(t_{n}\right)\right)-\delta_{t}^{-} \mathcal{F}\left(\psi^{n}\right)\right)\right]\right\|_{L^{2}}^{2}\right]
$$

In view of Lemma 3.2, the semi- $H^{1}$ norms on the RHS are equivalent to the discrete semi- $H^{1}$ norm of the corresponding grid functions, and we can estimate the RHS by estimating the corresponding discrete semi- $H^{1}$ norms, which has been essentially done in our recent work [4. For example, with the assumptions made in the claim (3.31), we have

$$
\begin{aligned}
& \delta_{x}^{+}\left(\mathcal{F}\left(\psi\left(x_{j}, t_{n}\right)\right)-\mathcal{F}\left(\psi_{j}^{n}\right)\right) \\
& =\int_{0}^{1}\left(G\left(\varsigma_{\theta}\left(t_{n}\right)\right) \delta_{x}^{+} \psi\left(x_{j}, t_{n}\right)+H\left(\varsigma_{\theta}\left(t_{n}\right)\right) \overline{\delta_{x}^{+} \psi\left(x_{j}, t_{n}\right)}\right) d \theta-\int_{0}^{1}\left(G\left(\varsigma_{\theta}^{n}\right) \delta_{x}^{+} \psi_{j}^{n}+H\left(\varsigma_{\theta}^{n}\right) \overline{\delta_{x}^{+} \psi_{j}^{n}}\right) d \theta \\
& =\int_{0}^{1}\left[\left(G\left(\varsigma_{\theta}\left(t_{n}\right)\right)-G\left(\varsigma_{\theta}^{n}\right)\right) \delta_{x}^{+} \psi\left(x_{j}, t_{n}\right)+\left(H\left(\varsigma_{\theta}\left(t_{n}\right)\right)-H\left(\varsigma_{\theta}^{n}\right)\right) \overline{\delta_{x}^{+} \psi\left(x_{j}, t_{n}\right)}\right] d \theta \\
& \left.+\int_{0}^{1}\left[G\left(\varsigma_{\theta}^{n}\right)\left(\delta_{x}^{+} \psi\left(x_{j}, t_{n}\right)-\delta_{x}^{+} \psi_{j}^{n}\right)+H\left(\varsigma_{\theta}^{n}\right) \overline{\left(\bar{\delta}_{x}^{+} \psi\left(x_{j}, t_{n}\right)\right.}-\overline{\delta_{x}^{+} \psi_{j}^{n}}\right)\right] d \theta, \quad j=0,1, \ldots, M-1,
\end{aligned}
$$

where $\varsigma_{\theta}\left(t_{n}\right)=\theta \psi\left(x_{j+1}, t_{n}\right)+(1-\theta) \psi\left(x_{j}, t_{n}\right)$ and $\varsigma_{\theta}^{n}=\theta \psi_{j+1}^{n}+(1-\theta) \psi_{j}^{n}$ for $\theta \in[0,1], H(z)$ and $G(z)$ are defined in (2.25). Using the global Lipschitz properties of $G(\cdot)$ and $H(\cdot)$ in the current case, then it is a direct consequence that [4]

$$
\left|\delta_{x}^{+}\left(\mathcal{F}\left(\psi\left(x_{j}, t_{n}\right)\right)-\mathcal{F}\left(\psi_{j}^{n}\right)\right)\right| \lesssim\left|\delta_{x}^{+}\left(\psi\left(x_{j}, t_{n}\right)-\psi_{j}^{n}\right)\right|+\sum_{k=j}^{j+1}\left|\psi\left(x_{k}, t_{n}\right)-\psi_{k}^{n}\right|, \quad 0 \leq j \leq M-1,
$$

which implies

$$
\left\|\delta_{x}^{+}\left(\mathcal{F}\left(\psi\left(x_{j}, t_{n}\right)\right)-\mathcal{F}\left(\psi_{j}^{n}\right)\right)\right\|_{l^{2}}^{2} \lesssim\left\|\delta_{x}^{+}\left(\psi\left(x_{j}, t_{n}\right)-\psi_{j}^{n}\right)\right\|_{l^{2}}^{2}+\left\|\psi\left(x_{j}, t_{n}\right)-\psi_{j}^{n}\right\|_{l^{2}}^{2} .
$$

Combining (A.2) together with Lemma 3.2, we have

$$
\begin{aligned}
\left\|\nabla\left[I_{M}\left(\mathcal{F}\left(\psi\left(t_{n}\right)\right)-\mathcal{F}\left(\psi^{n}\right)\right)\right]\right\|_{L^{2}}^{2} & \lesssim\left\|\nabla\left[I_{M}\left(\psi\left(t_{n}\right)-\psi^{n}\right)(\cdot)\right]\right\|_{L^{2}}^{2}+\left\|I_{M}\left(\psi\left(t_{n}\right)-\psi^{n}\right)(\cdot)\right\|_{L^{2}}^{2} \\
& \lesssim\left\|\nabla e^{n}(\cdot)\right\|_{L^{2}}^{2}+\left\|e^{n}(\cdot)\right\|_{L^{2}}^{2}+h^{2 m_{0}-2}
\end{aligned}
$$


The remaining term can be estimated in the same way and we omit the details here for brevity. Finally, we can obtain

$$
\frac{b-a}{2} \sum_{l=1}^{M-1}\left|\mu_{l}\right|^{2}\left|\widetilde{W}_{l}^{n, \pm}\right|^{2} \lesssim \tau^{2} \sum_{k=n-1}^{n}\left(\left\|\nabla e^{k}(\cdot)\right\|_{L^{2}}^{2}+\left\|e^{k}(\cdot)\right\|_{L^{2}}^{2}\right)+\tau^{2} h^{2 m_{0}-2}
$$

It is obvious that the constant in the inequality only depends on $f(\cdot), M_{1}$ and $\|\psi\|_{L^{\infty}\left([0, T] ; H_{s}^{m_{0}}\right)}$.

\section{REFERENCES}

[1] G. Akrivis, Finite difference discretization of the cubic Schrödinger equation, IMA J. Numer. Anal., 13 (1993), pp. $115-124$.

[2] W. Bao And Y. CAI, Optimal error estimates of finite difference methods for the Gross-Pitaevskii equation with angular momentum rotation, Math. Comp., 82 (2013), pp. 99-128.

[3] W. Bao and Y. CAI, Mathematical theory and numerical methods for Bose-Einstein condensation, Kinet. Relat. Mod., 6 (2013), pp. 1-135.

[4] W. BAO AND Y. CAI, Uniform error estimates of finite difference methods for the nonlinear Schrödinger equation with wave operator, SIAM J. Numer. Anal., 50 (2012), pp. 492-521.

[5] W. BAO AND X. Dong, Analysis and comparison of numerical methods for Klein-Gordon equation in nonrelativistic limit regime, Numer. Math., 120 (2012), pp. 189-229.

[6] W. BAO, X. Dong And J. XIN, Comparisons between sine-Gordon equation and perturbed nonlinear Schrödinger equations for modeling light bullets beyond critical collapse, Physica D, 239 (2010), pp. 1120-1134.

[7] W. Bao, D. Jaksch and P. A. Markowich, Numerical solution of the Gross-Pitaevskii equation for Bose-Einstein condensation, J. Comput. Phys., 187 (2003), pp. 318-342.

[8] L. Bergé And T. Colin, A singular perturbation problem for an enveloppe equation in plasma physics, Physica D, 84 (1995), pp. 437-459.

[9] C. Besse, B. Bidégaray and S. Descombes, Order estimates in time of splitting mehotds for the nonlinear Schrödinger equation, SIAM J. Numer. Anal., 40 (2002), pp. 26-40.

[10] Q. Chang, B. Guo And H. Jiang, Finite difference method for generalized Zakharov equations, Math. Comp., 64 (1995), pp. 537-553.

[11] T. Colin And P. Fabrie, Semidiscretization in time for Schrödinger-waves equations, Discrete Contin. Dyn. Syst., 4 (1998), pp. 671-690.

[12] A. Debussche And E. Faou, Modified energy for split-step methods applied to the linear Schrödinger equations, SIAM J. Numer. Anal., 47 (2009), pp. 3705-3719.

[13] P. Degond, J. Liu, M. Vignal, Analysis of an asymptotic preserving scheme for the Euler-Poisson system in the quasineutral limit, SIAM J. Numer. Anal., 46 (2008), pp. 1298-1322.

[14] W. Gautschi, Numerical integration of ordinary differential equations based on trigonometric polynomials, Numer. Math., 3 (1961), pp. 381-397.

[15] R. T. GLASSEY, Convergence of an energy-preserving scheme for the Zakharov equations in one space dimension, Math. Comp., 58 (1992), pp. 83-102.

[16] B. Guo And H. Liang, On the problem of numerical calculation for a class of systems of nonlinear Schrödinger equations with wave operator, J. Numer. Methods Comput. Appl., 4 (1983), pp. 176-182.

[17] R. H. HARdin And F. D. TAPperT, Applications of the split-step Fourier method to the numerical solution of nonlinear and variable coefficient wave equations, SIAM Rev. Chronicle, 15 (1973), pp. 423.

[18] M. Hochbruck And C. Lubich, A Gautschi-type method for oscillatory secondorder differential equations, Numer. Math., 83 (1999), pp. 403-426.

[19] S. Jin, Efficient Asymptotic-Preserving (AP) schemes for some multiscale kinetic equations, SIAM J. Sci. Comp., 21 (1999), pp. 441-454.

[20] H. O. Kreiss And J. Oliger, Stability of the Fourier method, SIAM J. Numer. Anal., 16 (1979), pp. 421-433.

[21] C. LuBICH, On splitting methods for Schrödinger-Poisson and cubic nonlinear Schrödinger equations, Math. Comp., 77 (2008), pp. 2141-2153.

[22] S. Machihara, K. NakAnishi and T. OzaWa, Nonrelativistic limit in the energy space for nonlinear Klein-Gordon equations, Math. Ann., 322 (2002), pp. 603-621.

[23] C. Neuhauser and M. Thalhammer, On the convergence of splitting methods for linear evolutionary Schrödinger equations involving an unbounded potential, BIT, 49 (2009), pp. 199-215.

[24] M. P. Robinson, G. Fairweather and B. M. Herbst, On the numerical solution of the cubic Schrödinger equation in one space variable, J. Comput. Phys., 104 (1993), pp. 277-284. 
[25] A. Y. Schoene, On the nonrelativistic limits of the Klein-Gordon and Dirac equations, J. Math. Anal. Appl., 71 (1979), pp. 36-47.

[26] J. Shen, T. TANG AND L. WANG, Spectral methods: algorithms, analysis and applications, Springer-Verlag, Berlin Heidelberg, 2011.

[27] T. R. TAha And M. J. Ablowitz, Analytical and numerical aspects of certain nonlinear evolution equations, II. Numerical, nonlinear Schrödinger equation, J. Comput. Phys., 55 (1984), pp. 203-230.

[28] V. ThомéE, Galerkin finite element methods for parabolic problems, Springer-Verlag, Berlin, Heidelberg, 1997.

[29] M. Tsutumi, Nonrelativistic approximation of nonlinear Klein-Gordon equations in two space dimensions, Nonlinear Analysis, 8 (1984), pp. 637-643.

[30] T. WAng And L. Zhang, Analysis of some new conservative schemes for nonlinear Schrödinger equation with wave operator, Appl. Math. Comput., 182 (2006), pp. 1780-1794.

[31] J. XIN, Modeling light bullets with the two-dimensional sine-Gordon equation, Physica D, 135 (2000), pp. 345-368. 